\title{
Positivity Preserving Interpolation Using Rational Bicubic Spline
}

\author{
Samsul Ariffin Abdul Karim, ${ }^{1}$ Kong Voon Pang, ${ }^{2}$ and Azizan Saaban ${ }^{3}$ \\ ${ }^{1}$ Department of Fundamental and Applied Sciences, Universiti Teknologi PETRONAS, Bandar Seri Iskandar, \\ 32610 Perak Darul Ridzuan, Malaysia \\ ${ }^{2}$ School of Mathematical Sciences, Universiti Sains Malaysia (USM), 11800 Minden, Penang, Malaysia \\ ${ }^{3}$ College of Arts and Sciences, Universiti Utara Malaysia (UUM), 06010 Sintok, Kedah, Malaysia
}

Correspondence should be addressed to Samsul Ariffin Abdul Karim; samsul_ariffin@petronas.com.my

Received 12 February 2015; Accepted 26 February 2015

Academic Editor: Juan Manuel Peña

Copyright (C) 2015 Samsul Ariffin Abdul Karim et al. This is an open access article distributed under the Creative Commons Attribution License, which permits unrestricted use, distribution, and reproduction in any medium, provided the original work is properly cited.

This paper discusses the positivity preserving interpolation for positive surfaces data by extending the $C^{1}$ rational cubic spline interpolant of Karim and Kong to the bivariate cases. The partially blended rational bicubic spline has 12 parameters in the descriptions where 8 of them are free parameters. The sufficient conditions for the positivity are derived on every four boundary curves network on the rectangular patch. Numerical comparison with existing schemes also has been done in detail. Based on Root Mean Square Error (RMSE), our partially blended rational bicubic spline is on a par with the established methods.

\section{Introduction}

Shape preserving interpolation is a process that involves some mathematical derivation to visualize the two-dimensional (2D) or three-dimensional (3D) data sets in the form of piecewise curves or surfaces that satisfies some degree of smoothness. Notably the shape preserving method will be able to maintain the original shape of the data sets. For example, if the given data is positive, then the final resulting curves or surface will be positive everywhere and it should be visual pleasing for the computer graphics displays. Usually the collected data from some laboratory experiment may contain little unwanted noise. Common cubic spline interpolation is not able to produce the shape preserving interpolation for positive, monotone and convexity of the data sets. Even though the interpolating curves are very smooth, that is, with $C^{2}$ continuity, there may exist some uncharacteristic behavior such as wiggle that will result in the negative values along the whole intervals. This unwanted flaw must be eliminated before the data are processed by the user. Therefore for the shape preserving interpolation and practical designing purposes, cubic spline and cubic Hermite spline are not recommended. Thus many researchers have proposed the idea of how to preserve the positive data by using some rational spline function with $C^{1}$ or $C^{2}$ continuity.

Some literature studies for positivity preserving are as follows. Abbas et al. [1,2] have discussed the positivity preserving by using partially blended rational bicubic spline function and rational bicubic spline function, respectively. Asim and Brodlie [3], Butt and Brodlie [4], and Brodlie et al. [5] discussed the use of bicubic spline for positivity preserving by inserting one or two extra knots in the region or interval where the negativity of the interpolant is found. Asim et al. [6] have studied the use of modified quadratic Shepard method for positivity preserving of large data sets. Duan et al. [7] have proposed new bivariate rational interpolation based on function values. They utilized the rational cubic spline of the forms of cubic numerator and linear denominator. Meanwhile, Duan et al. [8,9] investigated the bounded property, point control, and shape control of the bivariate rational cubic spline with linear denominator. Goodman et al. [10] discussed the constrained interpolation by using rational cubic with cubic denominator. Goodman [11] summarized all shape preserving criteria for the univariate cases. Hussain and Sarfraz [12] and M. Z. Hussain and M. Hussain [13] also studied the positivity preserving by utilizing the rational 
cubic spline of the forms cubic/cubic and cubic/quadratic, respectively. In the study by $M$. Z. Hussain and M. Hussain [13], there are no free parameters for the refining processes. M. Z. Hussain and M. Hussain [14] have developed the surface construction scheme for positivity preserving of positive scattered data arranged over triangular grids. They utilized the rational cubic spline of Tian et al. [15] without any free parameters. Hussain et al. [16] have developed shape preserving surfaces for positive and convex data using rational biquadratic splines. The degree of smoothness attained is $C^{0}$ as can clearly be seen from their graphical results. Ibraheem et al. [17] develop a positivity preserving scheme by using trigonometric rational functions which will increase the computation times. Karim and Kong [18] have proposed new rational cubic spline of the form cubic/quadratic with three parameters for local control of interpolating function. Positivity preserving by using this rational cubic spline has been addressed in the study by Karim and Kong [19]. Liu et al. [20] have studied the positivity and monotonicity preserving by using new rational biquartic spline by utilizing the extension cubic Ball basis function with two parameters. Saaban et al. [21] studied the positivity preserving by using quintic triangular Bezier patches. The surfaces are constructed by using convex combination of quintic triangular Bezier patches. They use the real data collected from the rainfall at various stations in West Peninsular of Malaysia. Sarfraz et al. [22] also have developed rational cubic spline scheme for positivity preserving of 3D data. Their scheme has 8 parameters without any free parameter. Furthermore Abbas et al. [1] indicated that Sarfraz et al. [22] works do not produce $C^{1}$ positive surfaces. The surfaces may only have $C^{0}$ continuity. BastianWalther and Schmidt [23] discussed the range restricted data interpolation for scattered data by using rational cubic spline of Gregory [24]. Wu et al. [25] studied the positivity preserving data approximation by using compactly supported radial basis functions (CSRBFs). Their scheme requires the solving of optimization problems in order to calculate the parameters that will guarantee that the positivity of the data is preserved. Peng et al. [26] have used the rational cubic spline of Hussain and Sarfraz [12] to construct the positive rational cubic Bezier interpolant for scattered positive data. Their methods require the modification of the first derivatives if the positivity of the rational interpolant is not met. The bicubic Hermite spline [27] interpolation cannot preserve the positivity of the surface data sets, since there maybe exist some negative values on the constructed surfaces. This paper is a continuation of our previous works. The univariate rational cubic spline interpolation in the studies by Karim and Kong $[18,19]$ is extended to the bivariate cases. Positivity preserving interpolation is our main object in this paper. We compare the performance between our rational bicubic spline and that of the work of Abbas et al. [1] by using Root Mean Square Error (RMSE).

We identify several advantages of our proposed partially blended rational bicubic spline interpolation for positive data as follows.

(i) Our schemes have 12 parameters and 8 of them are free parameters; meanwhile, in the study by M. Z.
Hussain and M. Hussain [13] the bivariate spline has 8 parameters and none of them are free parameters.

(ii) Our schemes are applicable for both equally or unequally spaced data; meanwhile, the rational cubic spline of Duan et al. [7-9] requires that the data be equally spaced.

(iii) The scheme can also be used whether the first derivative is given or not. Meanwhile, the works of Duan et al. $[7,9]$ require the true function values without the first derivative values.

(iv) Our rational bicubic spline is on a par with the works of Abbas et al. [1,2] and Hussain and Sarfraz [12]. Furthermore the presented scheme is easy to use and not involving any trigonometric functions compared with the work of Ibraheem et al. [17].

(v) When $\gamma_{i, j}=\widehat{\gamma}_{i, j}=0$, our partially blended rational bicubic spline is reduced to the work of M. Z. Hussain and M. Hussain [13].

(vi) The partially blended rational bicubic spline is unique and the free parameters in its description provide the flexibility in controlling the final shape of the positive surface data sets.

(vii) Furthermore based on Root Mean Square Error (RMSE) our partially blended rational bicubic spline gives numerical results as good as the numerical results from the study by Abbas et al. [1].

The remainder of the paper is organized as follows. Section 2 is devoted to the derivative estimation for $2 \mathrm{D}$ and $3 \mathrm{D}$ data by using Arithmetic Mean Method (AMM). Meanwhile, Section 3 reviews the univariate of rational cubic spline initiated by Karim and Kong [18, 19]. Positivity preserving for $2 \mathrm{D}$ data also is discussed in this section. The partially bicubic rational cubic spline interpolation is discussed in Section 4. Section 5 discusses the positivity preserving for 3D positive data sets by using the constructed partially bicubic rational cubic spline interpolation with numerical demonstrations with comparison to the existing schemes. Finally a summary and conclusions are given in Section 6.

\section{Derivative Estimation}

The first derivative that depends on the original data can be chosen by using Arithmetic Mean Method (AMM). The details are as follows:

For 2D Data

$$
\begin{gathered}
d_{0}=\Delta_{0}+\left(\Delta_{0}-\Delta_{1}\right)\left(\frac{h_{0}}{h_{0}+h_{1}}\right), \\
d_{n}=\Delta_{n-1}+\left(\Delta_{n-1}-\Delta_{n-2}\right)\left(\frac{h_{n-1}}{h_{n-1}+h_{n-2}}\right) .
\end{gathered}
$$

At the interior points, $x_{i}, i=1,2, \ldots, n-1$, the values of $d_{i}$ are given as

$$
d_{i}=\frac{h_{i-1} \Delta_{i}+h_{i} \Delta_{i-1}}{h_{i-1}+h_{i}} .
$$




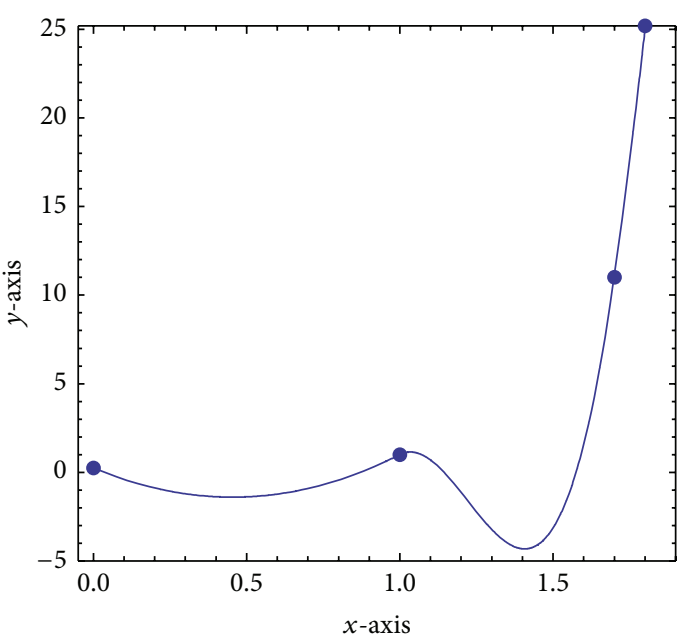

(a)

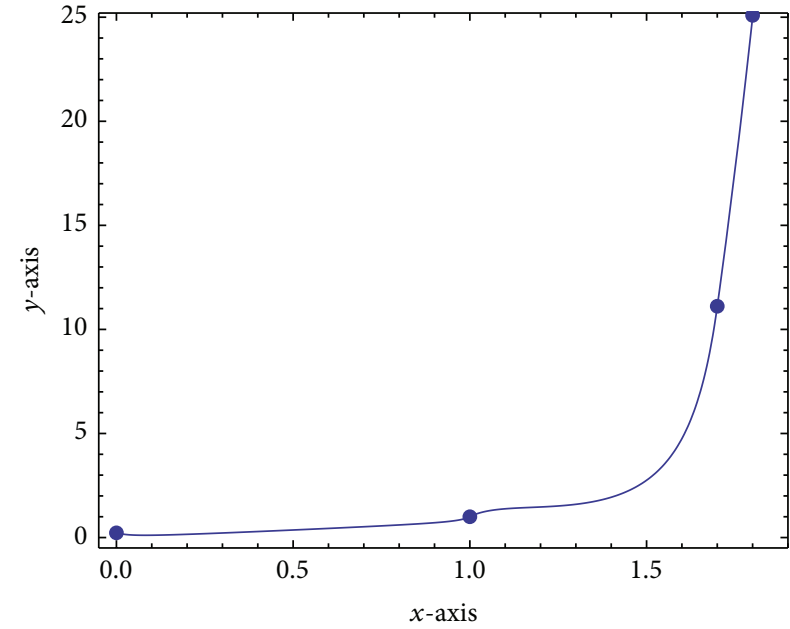

(b)

FIgURE 1: Comparison of (a) cubic Hermite spline curve and (b) our rational cubic spline with $\alpha_{i}=\beta_{i}=0.5$.

For 3D data

$$
\begin{gathered}
F_{0, j}^{x}=\Delta_{0, j}+\left(\Delta_{0, j}-\Delta_{1, j}\right) \frac{h_{1}}{\left(h_{1}+h_{2}\right)}, \\
F_{n, j}^{x}=\Delta_{n-1, j}+\left(\Delta_{n-1, j}-\Delta_{n-2, j}\right) \frac{h_{n-1}}{\left(h_{n-1}+h_{n-2}\right)}, \\
F_{i, j}^{x}=\frac{\Delta_{i, j}+\Delta_{i-1, j}}{2}, \quad i=1,2, \ldots, n-1, j=0,1, \ldots, m, \\
F_{i, m}^{y}=\widehat{\Delta}_{i, m-1}+\left(\widehat{\Delta}_{i, m-1}-\widehat{\Delta}_{i, m-2}\right) \frac{\widehat{h}_{m-1}}{\left(\widehat{h}_{m-1}+\widehat{h}_{m-2}\right)}, \\
F_{i, j}^{y}=\frac{\widehat{\Delta}_{i, j}+\left(\widehat{\Delta}_{i, 0}-\widehat{\Delta}_{i, 1}\right) \frac{\left.\widehat{\Delta}_{i, j-1}+\widehat{h}_{2}\right)}{2}, \quad i=0,1,2, \ldots, n, j=1,2, \ldots, m-1,}{\widehat{T}_{i, j}^{x y}=\frac{F_{i+1, j}^{y}-F_{i-1, j}^{y}}{h_{i-1}+h_{i}}+\frac{F_{i, j+1}^{x}-F_{i, j-1}^{x}}{\widehat{h}_{j-1}+\widehat{h}_{j}}} \\
i=1,2, \ldots, n-1, \quad j=1,2, \ldots, m-1,
\end{gathered}
$$

where

$$
\begin{array}{cc}
h_{i}=x_{i+1}-x_{i}, & \widehat{h}_{j}=y_{j+1}-y_{j}, \\
\Delta_{i, j}=\frac{f_{i+1}-f_{i}}{h_{i}}, & \widehat{\Delta}_{i, j}=\frac{f_{i, j+1}-f_{i, j}}{\widehat{h}_{j}} .
\end{array}
$$

TABle 1: Positive data from the study by Hussain et al. [28].

\begin{tabular}{ccccc}
\hline$i$ & 0 & 1 & 2 & 3 \\
\hline$x_{i}$ & 0.0 & 1.0 & 1.70 & 1.80 \\
\hline$f_{i}$ & 0.25 & 1.0 & 11.10 & 25 \\
\hline
\end{tabular}

TABLE 2: Numerical results.

\begin{tabular}{lcccc}
\hline$i$ & 0 & 1 & 2 & 3 \\
\hline$d_{i}$ & -7.296 & 8.796 & 123.429 & 154.570 \\
\hline$\Delta_{i}$ & 0.75 & 14.429 & 139 & \\
\hline$\alpha_{i}$ & 0.5 & 0.5 & 0.5 & \\
\hline$\beta_{i}$ & 0.5 & 0.5 & 0.5 & \\
\hline$\gamma_{i}$ & 13.84 & 3.14 & 0.25 & \\
\hline
\end{tabular}

\section{Review of Rational Cubic Spline Interpolant}

In this section the rational cubic spline of Karim and Kong $[18,19]$ is discussed briefly before it extends to the bivariate cases.

Given the set of data points $\left\{\left(x_{i}, f_{i}\right), i=0,1, \ldots, n\right\}$ such that $x_{0}<x_{1}<\cdots<x_{n}$, let $h_{i}=x_{i+1}-x_{i}, \Delta_{i}=\left(f_{i+1}-f_{i}\right) / h_{i}$, and $\theta=\left(x-x_{i}\right) / h_{i}$, where $0 \leq \theta \leq 1$. For $x \in\left[x_{i}, x_{i+1}\right]$, $i=0,1,2, \ldots, n-1$,

$$
s(x) \equiv s_{i}(x)=\frac{P_{i}(\theta)}{Q_{i}(\theta)},
$$

where

$$
\begin{gathered}
P_{i}(\theta)=A_{0}(1-\theta)^{3}+A_{1} \theta(1-\theta)^{2}+A_{2} \theta^{2}(1-\theta)+A_{3} \theta^{3}, \\
Q_{i}(\theta)=(1-\theta)^{2} \alpha_{i}+\theta(1-\theta)\left(2 \alpha_{i} \beta_{i}+\gamma_{i}\right)+\theta^{2} \beta_{i} .
\end{gathered}
$$


The rational function in (5) satisfies $C^{1}$ continuity as follows:

$$
\begin{gathered}
s\left(x_{i}\right)=f_{i}, \quad s\left(x_{i+1}\right)=f_{i+1}, \\
s_{i}^{(1)}\left(x_{i}\right)=d_{i}, \quad s_{i}^{(1)}\left(x_{i+1}\right)=d_{i+1} .
\end{gathered}
$$

It can be shown that the unknowns $A_{i}, i=0,1,2,3$, are given as follows:

$$
\begin{aligned}
& A_{0}=\alpha_{i} f_{i}, \\
& A_{1}=\left(2 \alpha_{i} \beta_{i}+\alpha_{i}+\gamma_{i}\right) f_{i}+\alpha_{i} h_{i} d_{i}, \\
& A_{2}=\left(2 \alpha_{i} \beta_{i}+\beta_{i}+\gamma_{i}\right) f_{i+1}-\beta_{i} h_{i} d_{i+1}, \\
& A_{3}=\beta_{i} f_{i+1},
\end{aligned}
$$

where $s_{i}^{(1)}(x)$ denotes derivative with respect to $x$ and $d_{i}$ denotes the derivative value which is given at the knot $x_{i}$, $i=0,1,2, \ldots, n$. The parameters are chosen such that $\alpha_{i}, \beta_{i}>$ $0, \gamma_{i} \geq 0$. When $\alpha_{i}=\beta_{i}=1, \gamma_{i}=0$, the rational cubic interpolant in (5) is reduced to cubic Hermite spline [19].

Positivity Preserving. For the strictly positive set of data $\left(x_{i}, f_{i}\right), i=0,1, \ldots, n, x_{0}<x_{1}<\cdots<x_{n}$,

$$
f_{i}>0, \quad i=0,1, \ldots, n .
$$

Karim and Kong [19] have developed the following results for strictly positive data sets given in (9).

Theorem 1. For strictly positive data defined in (9), the $C^{1}$ rational cubic interpolant defined over the interval $\left[x_{0}, x_{n}\right]$ is positive if in each subinterval $\left[x_{i}, x_{i+1}\right], i=0,1, \ldots, n-1$, the involving parameters $\alpha_{i}, \beta_{i}$, and $\gamma_{i}$ satisfy the following sufficient conditions:

$$
\begin{gathered}
\alpha_{i}, \beta_{i}>0, \\
\gamma_{i}=\lambda_{i}+\operatorname{Max}\left\{0,-\alpha_{i}\left[\frac{h_{i} d_{i}+\left(2 \beta_{i}+1\right) f_{i}}{f_{i}}\right],\right. \\
\left.\beta_{i}\left[\frac{h_{i} d_{i+1}-\left(2 \alpha_{i}+1\right) f_{i+1}}{f_{i+1}}\right]\right\}, \quad \lambda_{i}>0 .
\end{gathered}
$$

3.1. Numerical Demonstrations. In this section the positivity shape preserving using $C^{1}$ rational cubic spline of Karim and Kong [19] is discussed. The positive data is taken from the study by Hussain et al. [28].

Example 2. See Tables 1 and 2 and Figure 1.

\section{Rational Bicubic Spline Interpolant}

The piecewise rational cubic spline in (5) is extended to bicubic partially blended rational function $S(x, y)$ over rectangular domain $\Omega=[a, b] \times[c, d]$. The partition of the intervals $[a, b]$ and $[c, d]$ is defined as $\pi: a=x_{0}<x_{1}<\cdots<$ $x_{n}=b$ and $\bar{\pi}: c=y_{0}<y_{1}<\cdots<y_{m}=d$. The partially blended rational bicubic function over each rectangular patch $\left[x_{i}, x_{i+1}\right] \times\left[y_{j}, y_{j+1}\right], i=0,1, \ldots, n-1$ and $j=0,1, \ldots, m-1$, is defined as follows:

$$
S(x, y)=-A F B^{T}
$$

where

$$
\begin{aligned}
& F=\left(\begin{array}{ccc}
0 & S\left(x, y_{j}\right) & S\left(x, y_{j+1}\right) \\
S\left(x_{i}, y\right) & S\left(x_{i}, y_{j}\right) & S\left(x_{i}, y_{j+1}\right) \\
S\left(x_{i+1}, y\right) & S\left(x_{i+1}, y_{j}\right) & S\left(x_{i}, y_{j+1}\right)
\end{array}\right), \\
& A=\left[\begin{array}{lll}
-1 & a_{0}(\theta) & a_{1}(\theta)
\end{array}\right], \\
& B=\left[\begin{array}{lll}
-1 & b_{0}(\phi) & b_{1}(\phi)
\end{array}\right],
\end{aligned}
$$

where

$$
\begin{gathered}
a_{0}(\theta)=(1-\theta)^{2}(1+2 \theta), \\
a_{1}(\theta)=\theta^{2}(3-2 \theta), \\
b_{0}(\phi)=(1-\phi)^{2}(1+2 \phi), \\
b_{1}(\phi)=\phi^{2}(3-2 \phi), \\
\theta=\frac{x-x_{i}}{h_{i}}, \quad \phi=\frac{y-y_{j}}{\widehat{h}_{j}}, \\
h_{i}=x_{i+1}-x_{i}, \quad \widehat{h}_{j}=y_{j+1}-y_{j} .
\end{gathered}
$$

$S\left(x, y_{j}\right), S\left(x, y_{j+1}\right), S\left(x_{i}, y\right)$, and $S\left(x_{i+1}, y\right)$ are rational cubic functions (5) defined on the boundary of rectangular patch $\left[x_{i}, x_{i+1}\right] \times\left[y_{j}, y_{j+1}\right], i=0,1, \ldots, n-1, j=0,1, \ldots, m-1$, and are given as follows:

$$
S\left(x, y_{j}\right)=\frac{\sum_{i=0}^{3}(1-\theta)^{3-i} \theta^{i} A_{i}}{q_{1}(\theta)}
$$

with $A_{0}=\alpha_{i, j} F_{i, j}, A_{1}=\left(2 \alpha_{i, j} \beta_{i, j}+\alpha_{i, j}+\gamma_{i, j}\right) F_{i, j}+\alpha_{i, j} h_{i} F_{i, j}^{x}$, $A_{2}=\left(2 \alpha_{i, j} \beta_{i, j}+\beta_{i, j}+\gamma_{i, j}\right) F_{i+1, j}-\beta_{i, j} h_{i} F_{i+1, j}^{x}, A_{3}=\beta_{i, j} F_{i+1, j}$, and $q_{1}(\theta)=(1-\theta)^{2} \alpha_{i, j}+\left(2 \alpha_{i, j} \beta_{i, j}+\gamma_{i, j}\right) \theta(1-\theta)+\theta^{2} \beta_{i, j}$;

$$
S\left(x, y_{j+1}\right)=\frac{\sum_{i=0}^{3}(1-\theta)^{3-i} \theta^{i} B_{i}}{q_{2}(\theta)},
$$

with $B_{0}=\alpha_{i, j+1} F_{i, j+1}, B_{1}=\left(2 \alpha_{i, j+1} \beta_{i, j+1}+\alpha_{i, j+1}+\gamma_{i, j+1}\right) F_{i, j+1}+$ $\alpha_{i, j+1} h_{i} F_{i, j+1}^{x}, B_{2}=\left(2 \alpha_{i, j+1} \beta_{i, j+1}+\beta_{i, j+1}+\gamma_{i, j+1}\right) F_{i+1, j+1}-$ $\beta_{i, j+1} h_{i} F_{i+1, j+1}^{x}, B_{3}=\beta_{i, j} F_{i+1, j+1}$, and $q_{2}(\theta)=(1-\theta)^{2} \alpha_{i, j+1}+$ $\left(2 \alpha_{i, j+1} \beta_{i, j+1}+\gamma_{i, j+1}\right) \theta(1-\theta)+\theta^{2} \beta_{i, j+1}$;

$$
S\left(x_{i}, y\right)=\frac{\sum_{i=0}^{3}(1-\phi)^{3-i} \phi^{i} C_{i}}{q_{3}(\phi)},
$$

with $C_{0}=\widehat{\alpha}_{i, j} F_{i, j}, C_{1}=\left(2 \widehat{\alpha}_{i, j} \widehat{\beta}_{i, j}+\widehat{\alpha}_{i, j}+\widehat{\gamma}_{i, j}\right) F_{i, j}+\widehat{\alpha}_{i, j} \widehat{h}_{j} F_{i, j}^{y}$, $C_{2}=\left(2 \widehat{\alpha}_{i, j} \widehat{\beta}_{i, j}+\widehat{\beta}_{i, j}+\widehat{\gamma}_{i, j}\right) F_{i, j+1}-\widehat{\beta}_{i, j} \widehat{h}_{j} F_{i, j+1}^{y}, C_{3}=\widehat{\beta}_{i, j} F_{i, j+1}$, and $q_{3}(\phi)=(1-\phi)^{2} \widehat{\alpha}_{i, j}+\left(2 \widehat{\alpha}_{i, j} \widehat{\beta}_{i, j}+\widehat{\gamma}_{i, j}\right) \phi(1-\phi)+\phi^{2} \widehat{\beta}_{i, j}$;

$$
S\left(x_{i+1}, y\right)=\frac{\sum_{i=0}^{3}(1-\phi)^{3-i} \phi^{i} D_{i}}{q_{4}(\phi)},
$$




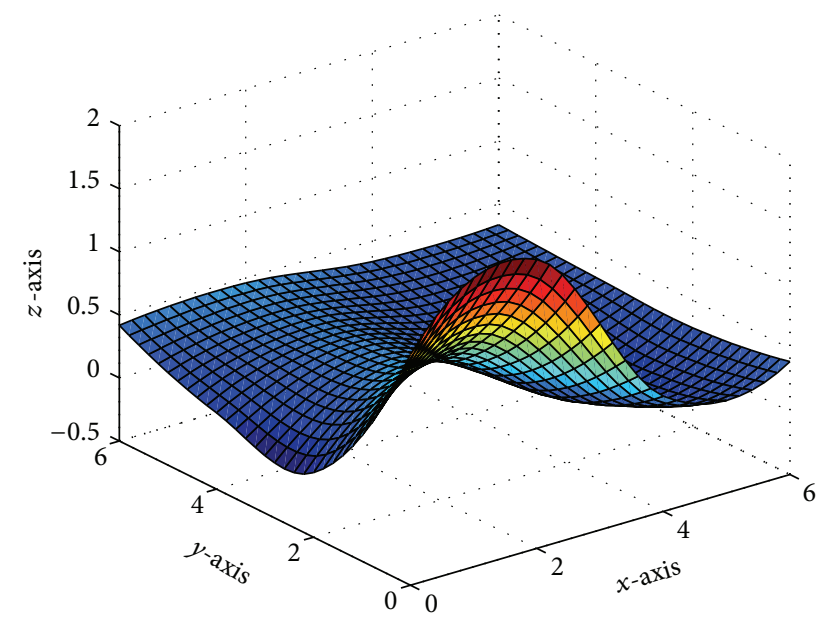

Figure 2: Bicubic Hermite surface.

with $D_{0}=\widehat{\alpha}_{i+1, j} F_{i+1, j}, D_{1}=\left(2 \widehat{\alpha}_{i+1, j} \widehat{\beta}_{i+1, j}+\widehat{\alpha}_{i+1, j}+\widehat{\gamma}_{i+1, j}\right) F_{i+1, j}+$ $\widehat{\alpha}_{i+1, j} \widehat{h}_{j} F_{i+1, j}^{y}, D_{2}=\left(2 \widehat{\alpha}_{i+1, j} \widehat{\beta}_{i+1, j}+\widehat{\beta}_{i+1, j}+\widehat{\gamma}_{i+1, j}\right) F_{i+1, j+1}-$ $\widehat{\beta}_{i+1, j} \widehat{h}_{j} F_{i+1, j+1}^{y}, D_{3}=\widehat{\beta}_{i, j} F_{i+1, j+1}$, and $q_{4}(\phi)=(1-\phi)^{2} \widehat{\alpha}_{i+1, j}+$ $\left(2 \widehat{\alpha}_{i+1, j} \widehat{\beta}_{i+1, j}+\widehat{\gamma}_{i+1, j}\right) \phi(1-\phi)+\phi^{2} \widehat{\beta}_{i+1, j}$.

Theorem 3. The partially blended rational bicubic spline $S(x, y)$ defined by (11) is $C^{1}$-continuous degree-seven piecewise rational surface.

Proof. The results trivially follow from Proposition 3 in the study by Casciola and Romani [29].

\section{Positivity Preserving of 3D Positive Data}

Let $\left(x_{i}, y_{i} F_{i, j}\right)$ be positive data defined over rectangular grid $\left[x_{i}, x_{i+1}\right] \times\left[y_{j}, y_{j+1}\right], i=0,1, \ldots, n-1, j=0,1, \ldots, m-1$, such that

$$
F_{i, j}>0, \quad \forall i, j
$$

Casciola and Romani [29] have developed the following results:

bicubic partially blended surface patch inherits all the properties of network of boundary curves.

Thus the partially bicubic surface patch in (21) is positive if boundary curves defined in (22)-(25) are positive; that is, $S\left(x, y_{j}\right)>0, S\left(x, y_{j+1}\right)>0, S\left(x_{i}, y\right)>0$, and $S\left(x_{i+1}, y\right)>0$, respectively. By simple algebraic manipulation we have the following theorem.

Theorem 4. The piecewise rational partially bicubic function $S(x, y)$ in (21) defined over the rectangular mesh $\left[x_{i}, x_{i+1}\right] \times$ $\left[y_{j}, y_{j+1}\right], i=0,1, \ldots, n-1, j=0,1, \ldots, m-1$, preserves
TABle 3: Positive surface data from function $F_{1}(x, y)$.

\begin{tabular}{lcccc}
\hline$y / x$ & 0 & 2 & 4 & 6 \\
\hline 0 & 1.33000 & 0.011261 & 0.10505 & 0.41710 \\
2 & 1.79240 & 0.619300 & 0.39739 & 0.45990 \\
4 & 0.41370 & 0.020814 & 0.16294 & 0.33635 \\
6 & 0.39537 & 0.281670 & 0.30087 & 0.33560 \\
\hline
\end{tabular}

the positive surface data if the parameters satisfy the following sufficient conditions:

$$
\begin{gathered}
\alpha_{i, j}>0, \quad \alpha_{i, j+1}>0, \quad \beta_{i, j}>0, \\
\beta_{i, j+1}>0, \quad \widehat{\alpha}_{i, j}>0, \quad \hat{\alpha}_{i+1, j}>0, \\
\hat{\beta}_{i, j}>0, \quad \hat{\beta}_{i+1, j}>0,
\end{gathered}
$$

$\gamma_{i, j}>\operatorname{Max}\left\{0, \frac{-\alpha_{i, j}\left(h_{i} F_{i, j}^{x}+\left(2 \beta_{i, j}+1\right) F_{i, j}\right)}{F_{i, j}}\right.$,

$$
\left.\frac{\beta_{i, j}\left(h_{i} F_{i+1, j}^{x}-\left(2 \alpha_{i, j}+1\right) F_{i+1, j}\right)}{F_{i+1, j}}\right\},
$$

$\gamma_{i, j+1}>\operatorname{Max}\left\{0, \frac{-\alpha_{i, j+1}\left(h_{i} F_{i, j+1}^{x}+\left(2 \beta_{i, j+1}+1\right) F_{i, j+1}\right)}{F_{i, j+1}}\right.$,

$$
\left.\frac{\beta_{i, j+1}\left(h_{i} F_{i+1, j+1}^{x}-\left(2 \alpha_{i, j+1}+1\right) F_{i+1, j+1}\right)}{F_{i+1, j+1}}\right\},
$$

$$
\widehat{\gamma}_{i, j}>\operatorname{Max}\left\{0, \frac{-\widehat{\alpha}_{i, j}\left(\widehat{h}_{j} F_{i, j}^{y}+\left(2 \widehat{\beta}_{i, j}+1\right) F_{i, j}\right)}{F_{i, j}},\right.
$$

$$
\left.\frac{\widehat{\beta}_{i, j}\left(\widehat{h}_{j} F_{i, j+1}^{y}-\left(2 \widehat{\alpha}_{i, j}+1\right) F_{i, j+1}\right)}{F_{i, j+1}}\right\}
$$

$$
\begin{aligned}
\widehat{\gamma}_{i+1, j}>\operatorname{Max}\left\{0, \frac{-\widehat{\alpha}_{i+1, j}\left(\widehat{h}_{j} F_{i+1, j}^{y}+\left(2 \widehat{\beta}_{i+1, j}+1\right) F_{i+1, j}\right)}{F_{i+1, j}},\right. & \left.\frac{\widehat{\beta}_{i+1, j}\left(\widehat{h}_{j} F_{i+1, j+1}^{y}-\left(2 \widehat{\alpha}_{i+1, j}+1\right) F_{i+1, j+1}\right)}{F_{i+1, j+1}}\right\} .
\end{aligned}
$$

Proof. From (14)-(17), $S\left(x, y_{j}\right)>0$, if $A_{i}>0, i=0,1,2,3$. Now for $\alpha_{i, j}>0, \beta_{i, j}>0$, and $\gamma_{i, j}>0, S\left(x, y_{j}\right)>0$, if

$$
\begin{array}{r}
\gamma_{i, j}>\frac{-\alpha_{i, j}\left(h_{i} F_{i, j}^{x}+\left(2 \beta_{i, j}+1\right) F_{i, j}\right)}{F_{i, j}}, \\
\gamma_{i, j}>\frac{\beta_{i, j}\left(h_{i} F_{i+1, j}^{x}-\left(2 \alpha_{i, j}+1\right) F_{i+1, j}\right)}{F_{i+1, j}} .
\end{array}
$$




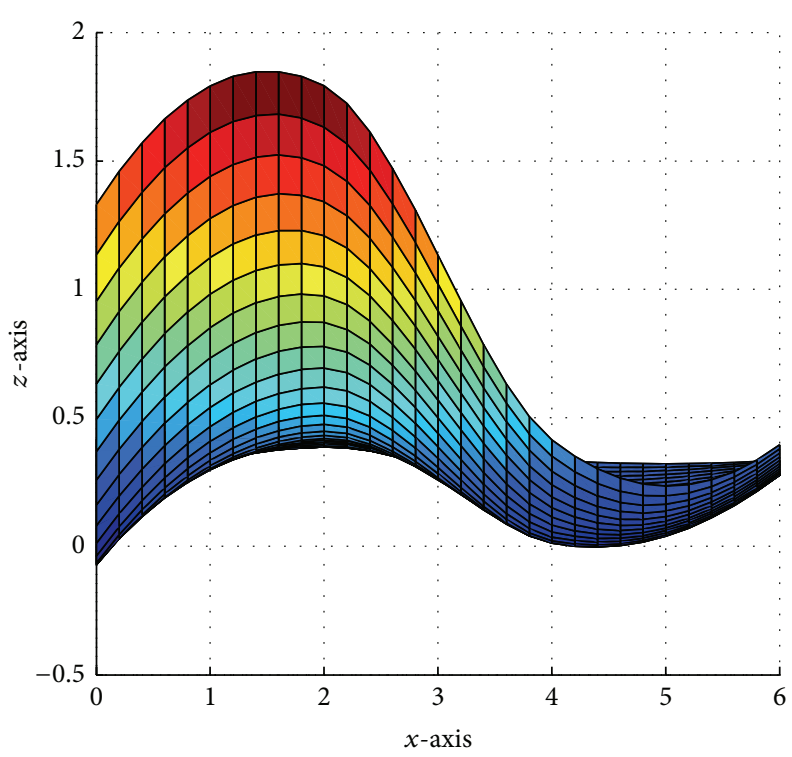

(a)

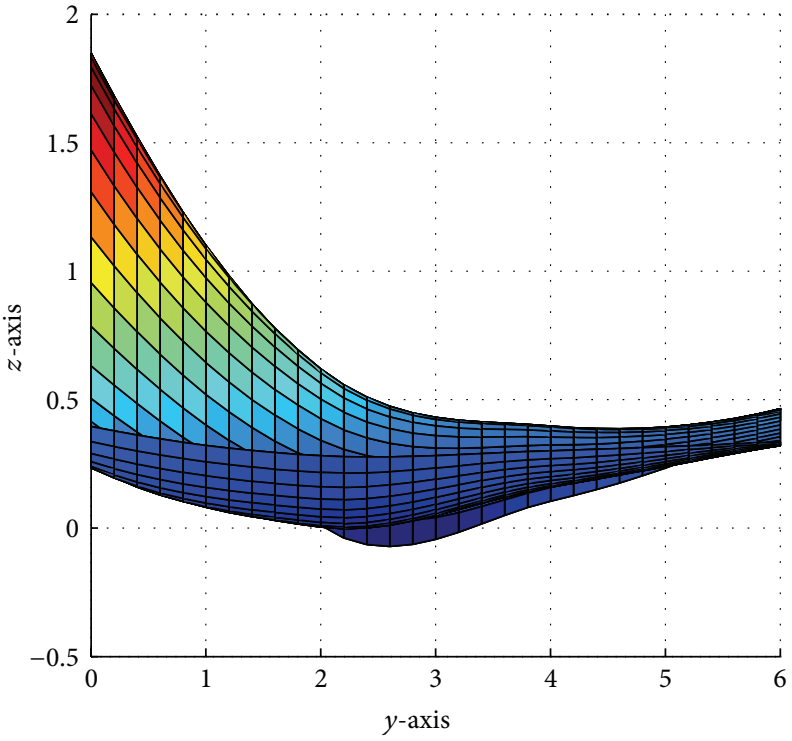

(b)

Figure 3: Bicubic Hermite surface: (a) $x z$-view and (b) $y z$-view for Figure 2, respectively.

Combining the conditions in (20) results in the following sufficient conditions for the positivity of $\left(x, y_{j}\right)$ :

$$
\begin{aligned}
\gamma_{i, j}>\operatorname{Max}\left\{0, \frac{-\alpha_{i, j}\left(h_{i} F_{i, j}^{x}+\left(2 \beta_{i, j}+1\right) F_{i, j}\right)}{F_{i, j}},\right. & \left.\frac{\beta_{i, j}\left(h_{i} F_{i+1, j}^{x}-\left(2 \alpha_{i, j}+1\right) F_{i+1, j}\right)}{F_{i+1, j}}\right\} .
\end{aligned}
$$

Likewise the remaining three boundary curves $S\left(x, y_{j+1}\right)$, $S\left(x_{i}, y\right)$, and $S\left(x_{i+1}, y\right)$ are positive if the following sufficient conditions are satisfied:

$$
\begin{gathered}
\gamma_{i, j+1}>\frac{-\alpha_{i, j+1}\left(h_{i} F_{i, j+1}^{x}+\left(2 \beta_{i, j+1}+1\right) F_{i, j+1}\right)}{F_{i, j+1}}, \\
\gamma_{i, j+1}>\frac{\beta_{i, j+1}\left(h_{i} F_{i+1, j+1}^{x}-\left(2 \alpha_{i, j+1}+1\right) F_{i+1, j+1}\right)}{F_{i+1, j+1}}, \\
\widehat{\gamma}_{i, j}>\frac{-\widehat{\alpha}_{i, j}\left(\widehat{h}_{j} F_{i, j}^{y}+\left(2 \widehat{\beta}_{i, j}+1\right) F_{i, j}\right)}{F_{i, j}}, \\
\widehat{\gamma}_{i, j}>\frac{\widehat{\beta}_{i, j}\left(\widehat{h}_{j} F_{i, j+1}^{y}-\left(2 \widehat{\alpha}_{i, j}+1\right) F_{i, j+1}\right)}{F_{i, j+1}}, \\
\widehat{\gamma}_{i+1, j}>\frac{-\widehat{\alpha}_{i+1, j}\left(\widehat{h}_{j} F_{i+1, j}^{y}+\left(2 \widehat{\beta}_{i+1, j}+1\right) F_{i+1, j}\right)}{F_{i+1, j}}, \\
\widehat{\gamma}_{i+1, j}>\frac{\widehat{\beta}_{i+1, j}\left(\widehat{h}_{j} F_{i+1, j+1}^{y}-\left(2 \widehat{\alpha}_{i+1, j}+1\right) F_{i+1, j+1}\right)}{F_{i+1, j+1}} .
\end{gathered}
$$

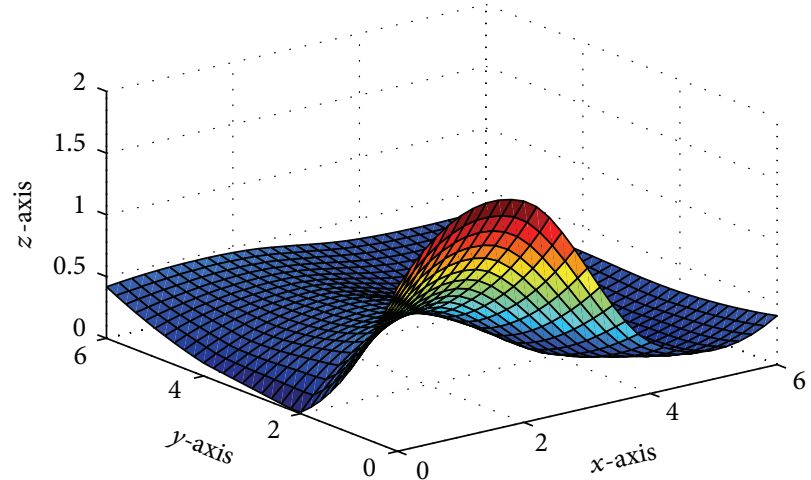

FIgURE 4: Positivity preserving by using our partially rational bicubic spline.

Combining the conditions in (22) and (23), (24) and (25), and (26) and (27) leads us to the following:

$$
\begin{gathered}
\gamma_{i, j+1}>\operatorname{Max}\left\{0, \frac{-\alpha_{i, j+1}\left(h_{i} F_{i, j+1}^{x}+\left(2 \beta_{i, j+1}+1\right) F_{i, j+1}\right)}{F_{i, j+1}},\right. \\
\left.\frac{\beta_{i, j+1}\left(h_{i} F_{i+1, j+1}^{x}-\left(2 \alpha_{i, j+1}+1\right) F_{i+1, j+1}\right)}{F_{i+1, j+1}}\right\}, \\
\widehat{\gamma}_{i, j}>\operatorname{Max}\left\{\begin{array}{l}
0, \frac{-\widehat{\alpha}_{i, j}\left(\widehat{h}_{j} F_{i, j}^{y}+\left(2 \widehat{\beta}_{i, j}+1\right) F_{i, j}\right)}{F_{i, j}}, \\
\left.\frac{\widehat{\beta}_{i, j}\left(\widehat{h}_{j} F_{i, j+1}^{y}-\left(2 \widehat{\alpha}_{i, j}+1\right) F_{i, j+1}\right)}{F_{i, j+1}}\right\},
\end{array}\right.
\end{gathered}
$$




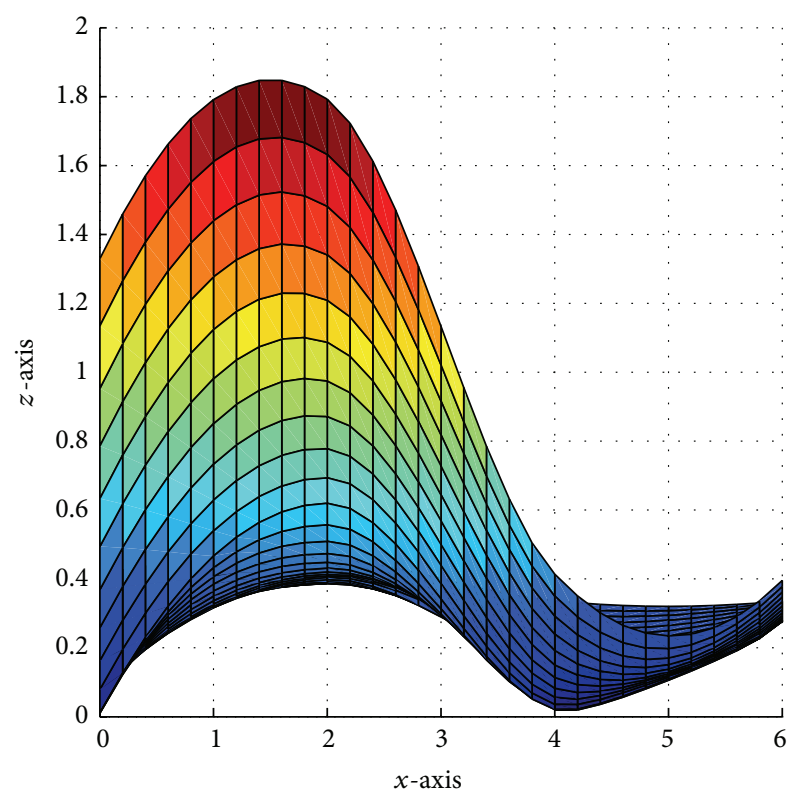

(a)

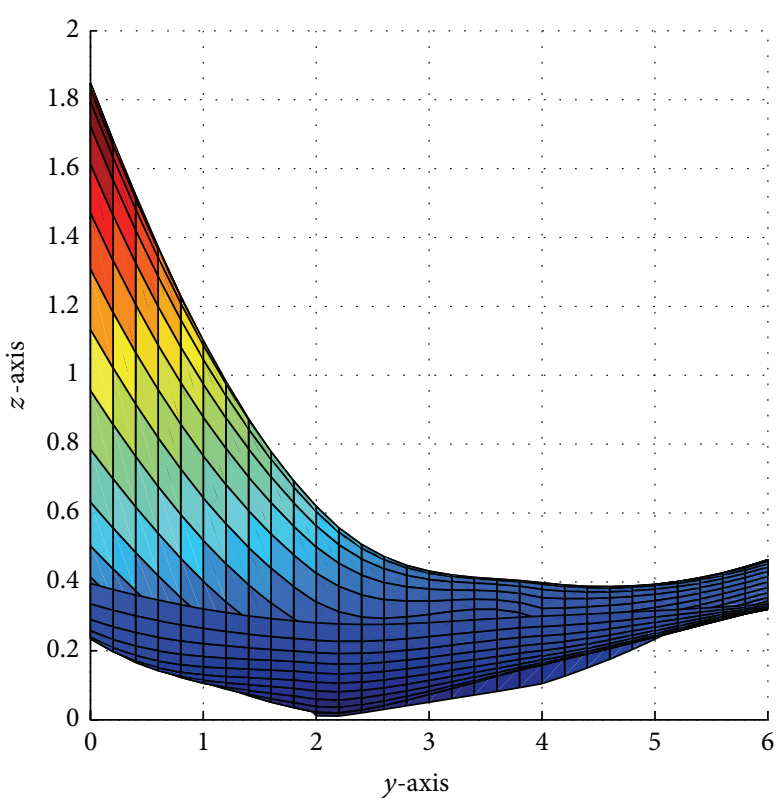

(b)

FIGURE 5: Positivity preserving surface: (a) $x z$-view and (b) $y z$-view for Figure 4, respectively.

TABLE 4: Positive surface data from function $F_{2}(x, y)$.

\begin{tabular}{lcccccc}
\hline$y / x$ & -3 & -2 & -1 & 1 & 2 \\
\hline-3 & 1.536600 & 0.37930 & 0.055529 & 1.9445 & 1.62070 & 1.80150 \\
-2 & 1.175100 & 0.19847 & 0.106150 & 1.8939 & 1.4108 & 0.25095 \\
-1 & 0.044919 & 1.74900 & 0.589220 & 1.3596 & 1.67110 & 1.95510 \\
1 & 0.107150 & 0.32885 & 0.640360 & 1.1349 & 1.26740 & 1.89290 \\
2 & 0.605060 & 0.73262 & 0.865080 & 1.0498 & 1.09940 \\
3 & 0.851190 & 0.90059 & 0.950230 & 1.39490 \\
\hline
\end{tabular}

TABLe 5: Positive surface data from function $F_{3}(x, y)$.

\begin{tabular}{lccccccc}
\hline$y / x$ & -3 & -2 & -1 & 0 & 1 & 2 \\
\hline-3 & 0.0401 & 0.0404 & 0.1755 & 1.0401 & 0.1755 & 0.0404 & 0.0401 \\
-2 & 0.0583 & 0.0586 & 0.1936 & 1.0583 & 0.1936 & 0.0586 & 0.0583 \\
-1 & 0.4078 & 0.4082 & 0.5432 & 1.4079 & 0.5432 & 0.4082 \\
0 & 1.0400 & 1.0403 & 1.1753 & 2.0400 & 1.1753 & 1.0403 & 0.4078 \\
1 & 0.4078 & 0.4082 & 0.5432 & 1.4079 & 0.5432 & 0.4082 & 0.4078 \\
2 & 0.0583 & 0.0586 & 0.1936 & 1.0583 & 0.1936 & 0.0586 \\
3 & 0.0401 & 0.0404 & 0.1755 & 1.0401 & 0.1755 & 0.0404 & 0.0583 \\
\hline
\end{tabular}

TABle 6: Positive surface data from function $F_{4}(x, y)$.

\begin{tabular}{lccccrr}
\hline$y / x$ & -3 & -2 & -1 & 1 & 2 & 0.0238 \\
\hline-3 & 0.0124 & 0.0238 & 0.0404 & 0.0404 & 0.1667 & 0.0635 \\
-2 & 0.0238 & 0.0635 & 0.1667 & 1.3333 & 0.1667 & 0.0238 \\
-1 & 0.0404 & 0.1667 & 1.3333 & 1.3333 & 0.1667 & 0.0404 \\
1 & 0.0404 & 0.1667 & 1.3333 & 0.1667 & 0.0635 & 0.0404 \\
2 & 0.0238 & 0.0635 & 0.1667 & 0.0404 & 0.0238 \\
3 & 0.0124 & 0.0238 & 0.0404 & & 0.0124 \\
\hline
\end{tabular}




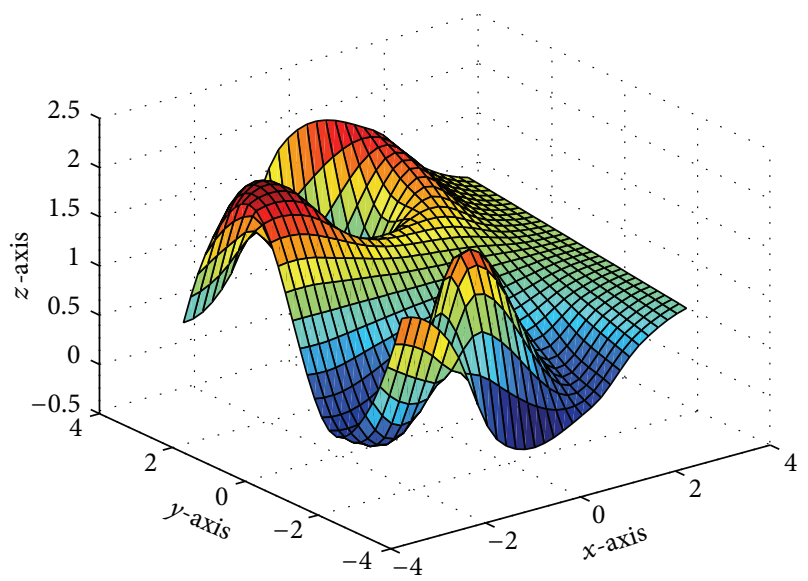

FIgURE 6: Bicubic Hermite surface.

$$
\begin{aligned}
& \widehat{\gamma}_{i+1, j}>\operatorname{Max}\left\{0, \frac{-\widehat{\alpha}_{i+1, j}\left(\widehat{h}_{j} F_{i+1, j}^{y}+\left(2 \widehat{\beta}_{i+1, j}+1\right) F_{i+1, j}\right)}{F_{i+1, j}},\right. \\
& \left.\frac{\widehat{\beta}_{i+1, j}\left(\widehat{h}_{j} F_{i+1, j+1}^{y}-\left(2 \widehat{\alpha}_{i+1, j}+1\right) F_{i+1, j+1}\right)}{F_{i+1, j+1}}\right\} \text {. }
\end{aligned}
$$

Then from (21) and (28), sufficient conditions for the positivity of network of all four curves $S\left(x, y_{j}\right), S\left(x, y_{j+1}\right), S\left(x_{i}, y\right)$, and $S\left(x_{i+1}, y\right)$ are obtained. Thus the proof is complete.

The sufficient conditions in (19) can be rewritten as

$$
\begin{gathered}
\alpha_{i, j}>0, \quad \alpha_{i, j+1}>0, \quad \beta_{i, j}>0, \\
\beta_{i, j+1}>0, \quad \widehat{\alpha}_{i, j}>0, \quad \widehat{\alpha}_{i+1, j}>0, \\
\widehat{\beta}_{i, j}>0, \quad \widehat{\beta}_{i+1, j}>0, \\
\gamma_{i, j}=k_{i, j}+\operatorname{Max}\left\{0, \frac{-\alpha_{i, j}\left(h_{i} F_{i, j}^{x}+\left(2 \beta_{i, j}+1\right) F_{i, j}\right)}{F_{i, j}},\right. \\
\left.\frac{\beta_{i, j}\left(h_{i} F_{i+1, j}^{x}-\left(2 \alpha_{i, j}+1\right) F_{i+1, j}\right)}{F_{i+1, j}}\right\},
\end{gathered}
$$

$\gamma_{i, j+1}=l_{i, j}$

$$
\begin{array}{r}
+\operatorname{Max}\left\{0, \frac{-\alpha_{i, j+1}\left(h_{i} F_{i, j+1}^{x}+\left(2 \beta_{i, j+1}+1\right) F_{i, j+1}\right)}{F_{i, j+1}},\right. \\
\left.\frac{\beta_{i, j+1}\left(h_{i} F_{i+1, j+1}^{x}-\left(2 \alpha_{i, j+1}+1\right) F_{i+1, j+1}\right)}{F_{i+1, j+1}}\right\}, \\
\widehat{\gamma}_{i, j}=m_{i, j}+\operatorname{Max}\left\{0, \frac{-\widehat{\alpha}_{i, j}\left(\widehat{h}_{j} F_{i, j}^{y}+\left(2 \widehat{\beta}_{i, j}+1\right) F_{i, j}\right)}{F_{i, j}},\right. \\
\left.\frac{\widehat{\beta}_{i, j}\left(\widehat{h}_{j} F_{i, j+1}^{y}-\left(2 \widehat{\alpha}_{i, j}+1\right) F_{i, j+1}\right)}{F_{i, j+1}}\right\},
\end{array}
$$

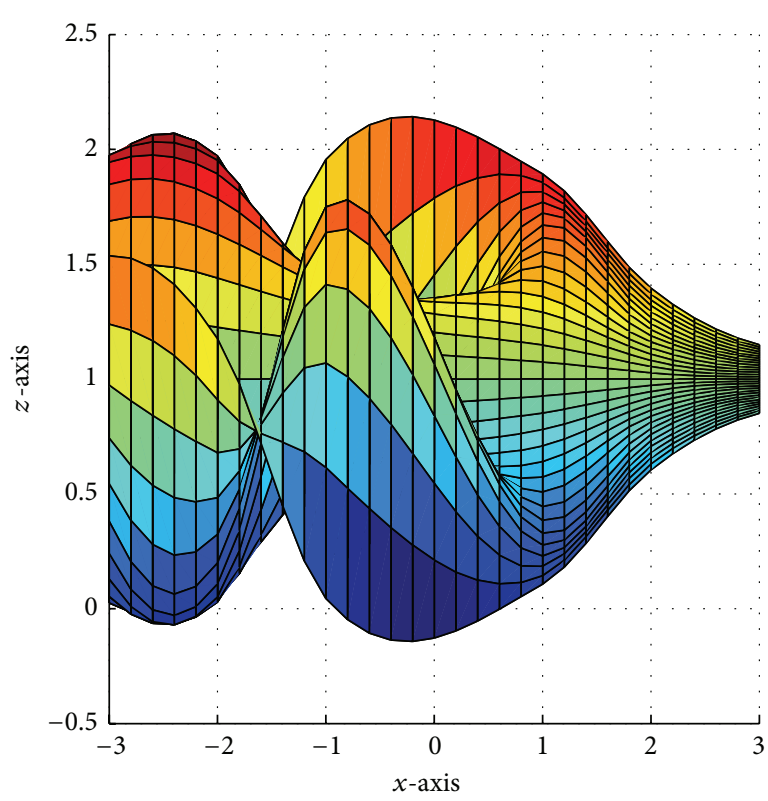

(a)

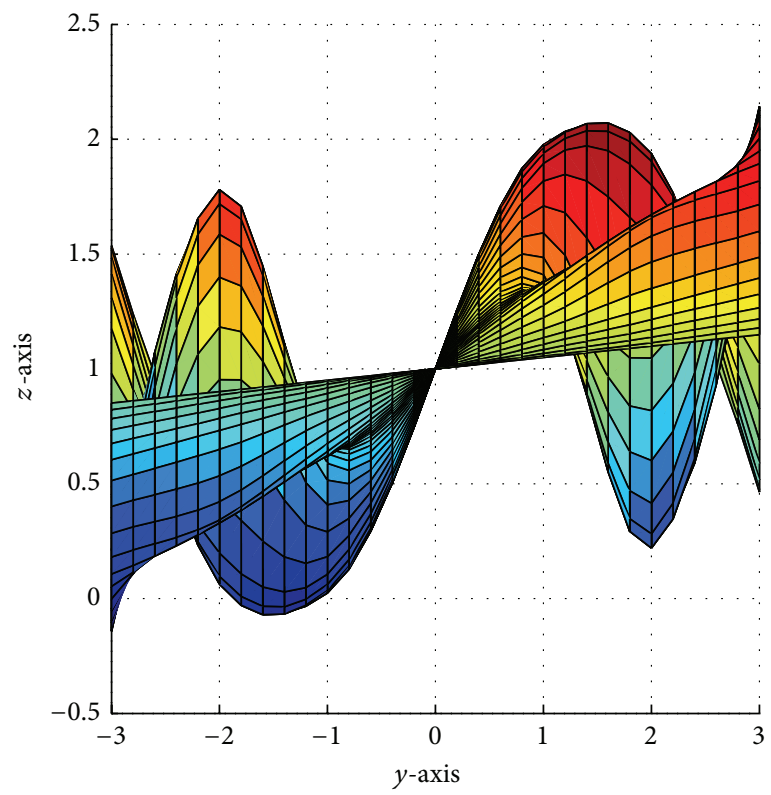

(b)

FIgure 7: Bicubic Hermite surface: (a) $x z$-view and (b) $y z$-view for Figure 6, respectively.

$$
\begin{aligned}
& \widehat{\gamma}_{i+1, j}=n_{i, j} \\
& +\operatorname{Max}\left\{0, \frac{-\widehat{\alpha}_{i+1, j}\left(\widehat{h}_{j} F_{i+1, j}^{y}+\left(2 \widehat{\beta}_{i+1, j}+1\right) F_{i+1, j}\right)}{F_{i+1, j}},\right. \\
& \left.\frac{\widehat{\beta}_{i+1, j}\left(\widehat{h}_{j} F_{i+1, j+1}^{y}-\left(2 \widehat{\alpha}_{i+1, j}+1\right) F_{i+1, j+1}\right)}{F_{i+1, j+1}}\right\},
\end{aligned}
$$

where $k_{i, j}>0, l_{i, j}>0, m_{i, j}>0$, and $n_{i, j}>0$. 


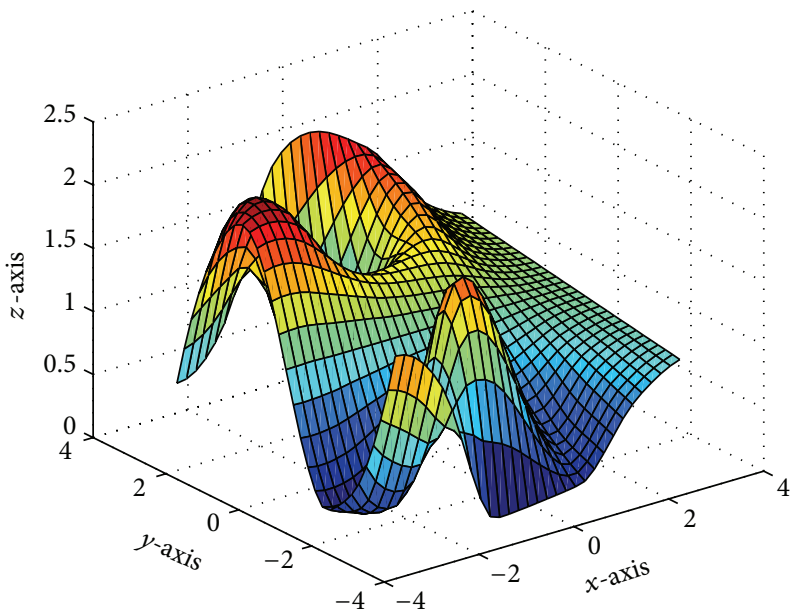

FIGURE 8: Positivity preserving by using our partially rational bicubic spline.

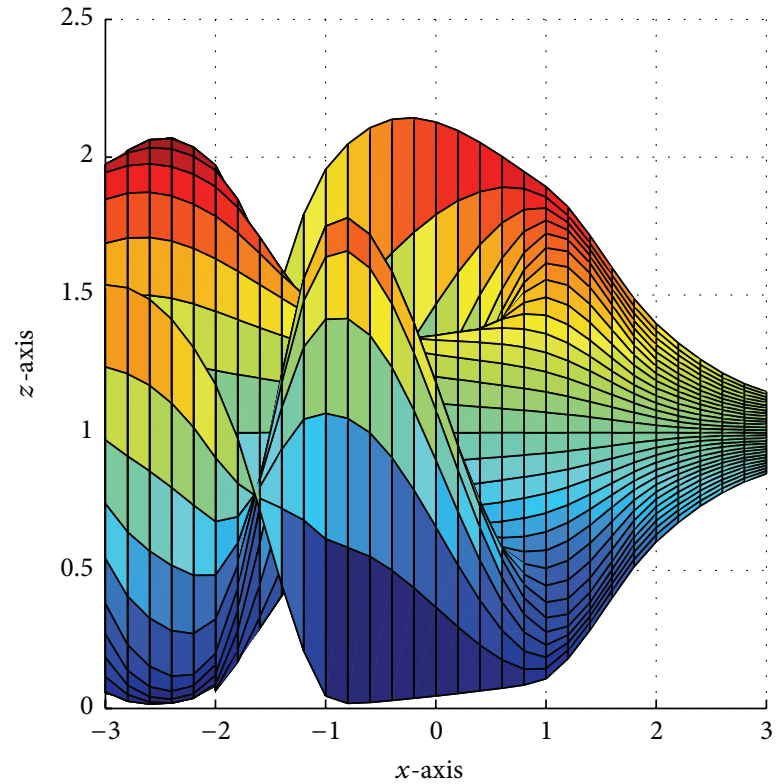

(a)

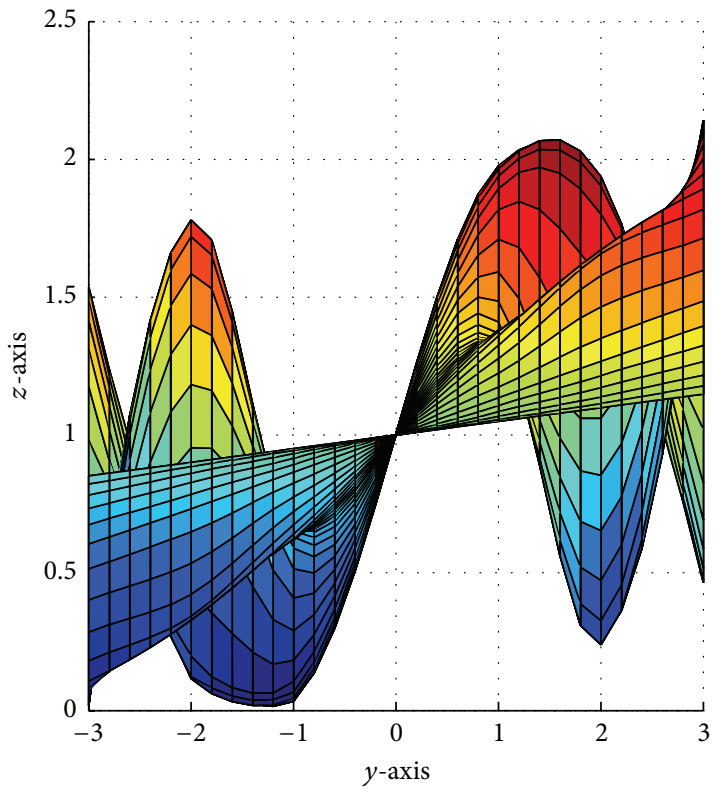

(b)

FIGURE 9: Positivity preserving surface: (a) $x z$-view and (b) $y z$-view for Figure 8, respectively.

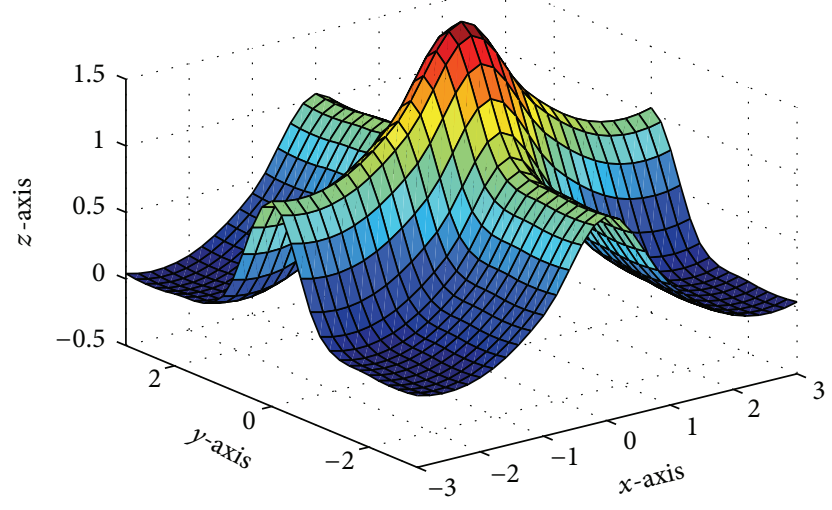

FIGURE 10: Bicubic Hermite surface. 


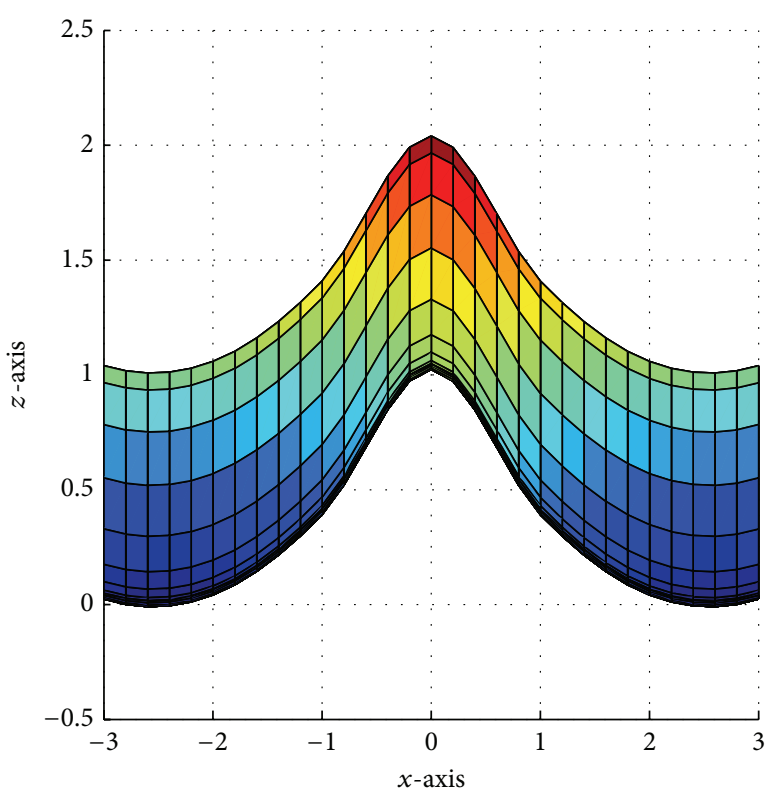

(a)

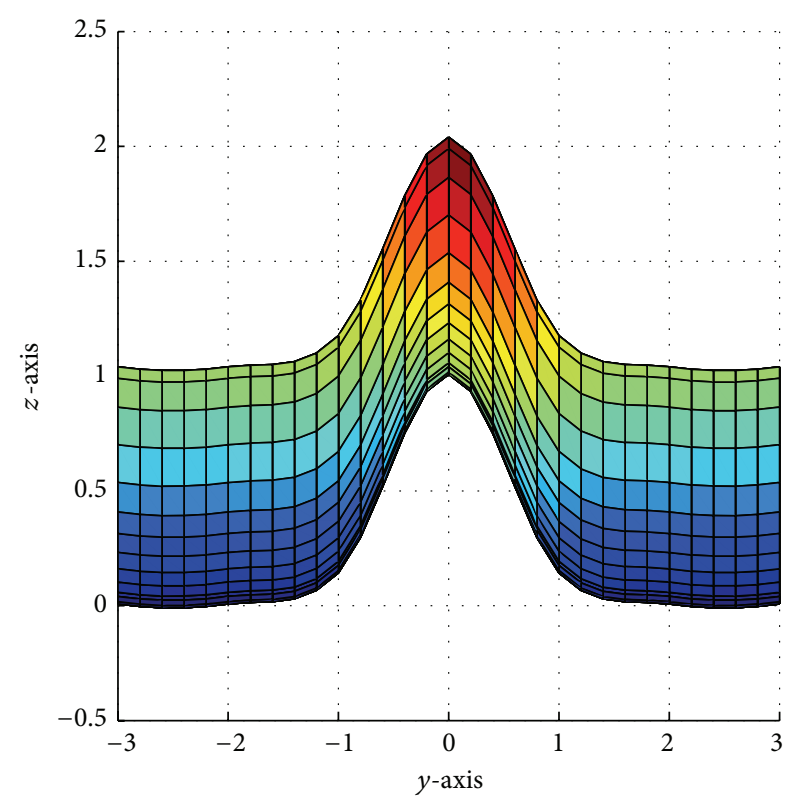

(b)

Figure 11: Bicubic Hermite surface: (a) $x z$-view and (b) $y z$-view for Figure 10, respectively.

Theorem 5. The partially blended rational bicubic spline $S(x, y)$ constructed by using shape parameters calculated from sufficient condition for positivity in (29) is $C^{1}$-continuous degree-seven piecewise rational surface with positivity preserving properties to the positive data sets.

Proof. The proof also trivially follows from the work of Casciola and Romani [29].

5.1. Algorithm for Computer Implementations. The algorithm is for the purpose of computer implementation of positivity preserving by using our partially rational bicubic spline presented in Theorem 4.

Input. The input is the positive data points $\left(x_{i}, y_{j}, F_{i, j}\right), i=$ $0,1, \ldots, n$ and $j=0,1, \ldots, m$.

Output. The output is the positive surfaces.

Step 1. Calculate the first derivative values $F_{i, j}^{x}, F_{i, j}^{y}, F_{i, j}^{x y}$ by using AMM.

Step 2. Choose suitable values for free parameters $\alpha_{i, j}, \beta_{i, j}$, $\alpha_{i, j+1}, \beta_{i, j+1}, \widehat{\alpha}_{i, j}, \widehat{\alpha}_{i+1, j}, \widehat{\beta}_{i, j}, \widehat{\beta}_{i+1, j}$ and $k_{i, j}, l_{i, j}, m_{i, j}, n_{i, j}$, and compute the values of parameters $\gamma_{i, j}, \gamma_{i, j+1}, \widehat{\gamma}_{i, j}, \widehat{\gamma}_{i+1, j}$ by using Theorem 4.

Step 3. Construct the positive surfaces by substituting all the required parameters into the partially blended rational bicubic spline function (11).

Repeat Steps 2 and 3 to generate different positive surfaces through different positive data sets.

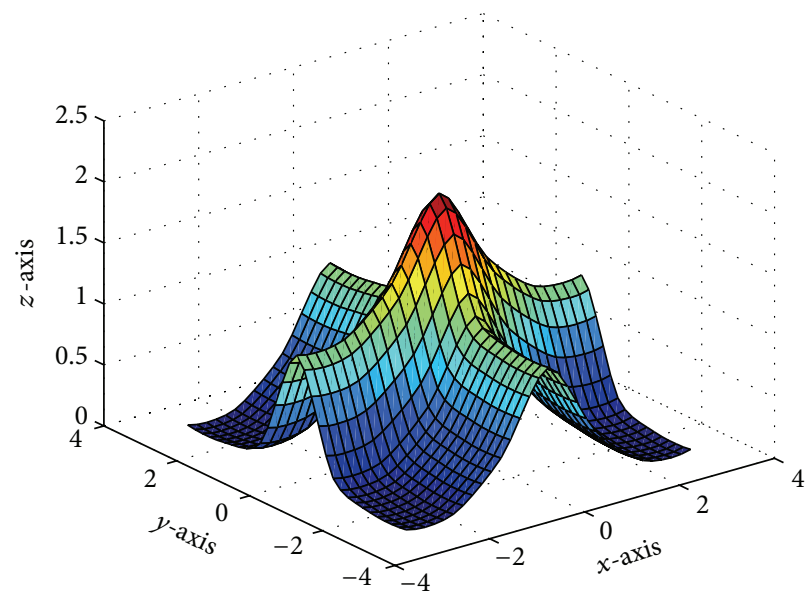

FIGURE 12: Positivity preserving by using our partially rational bicubic spline.

5.2. Numerical Demonstrations. In this section, numerical examples for positivity preserving by using partially blended rational bicubic spline interpolation of positive surface data will be discussed in detail. Four positive data sets are taken from the studies by Abbas et al. $[1,2]$ and Hussain and Sarfraz [12], respectively.

Example 6. Positive data from the following function is truncated to five decimal places [1]:

$$
\begin{array}{r}
F_{1}(x, y)=e^{-\left(x^{2}+y^{2}\right) / 15}(\sin (x)+\cos (y))+0.33 \\
0 \leq x, y \leq 6 .
\end{array}
$$




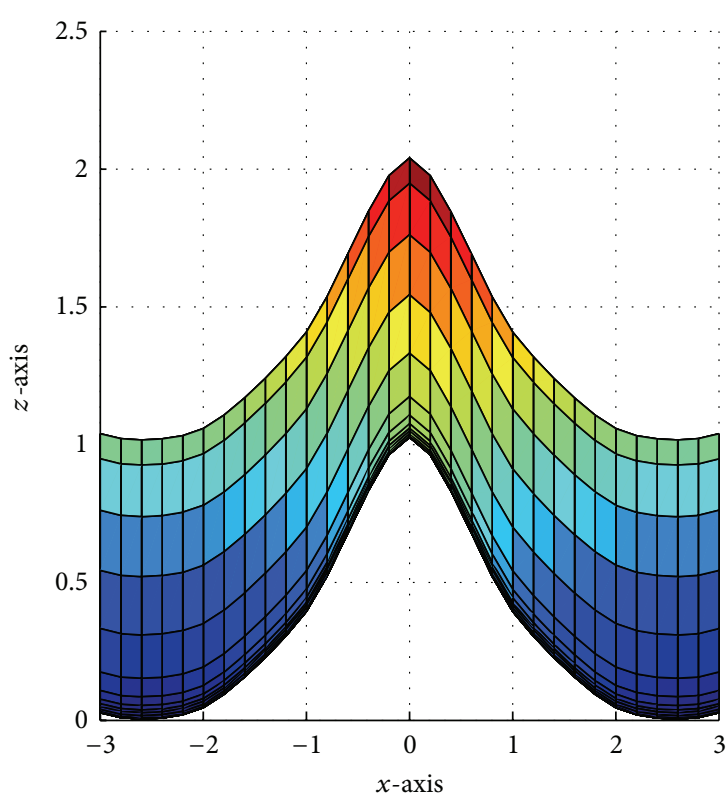

(a)

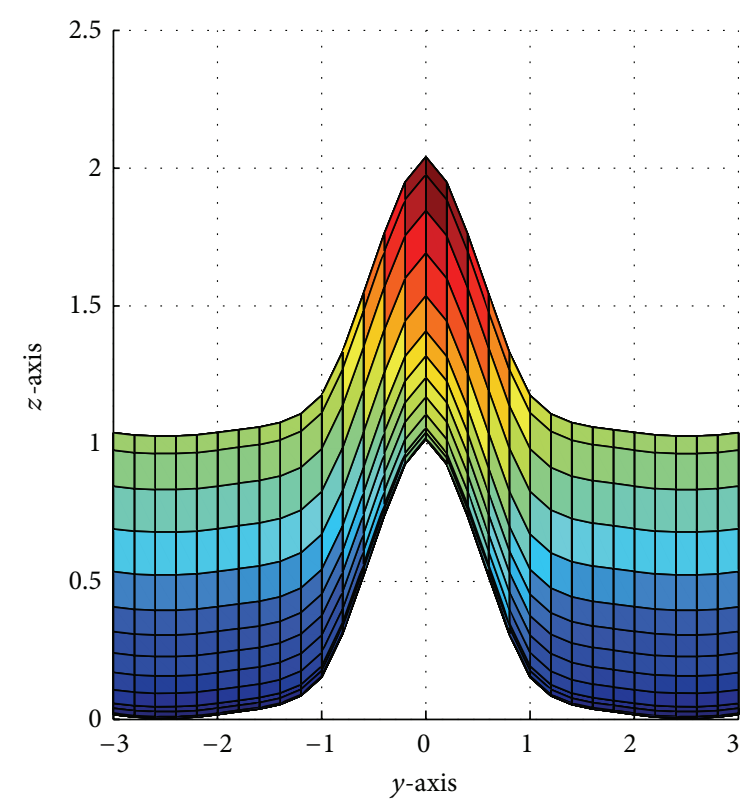

(b)

FIgURE 13: Positivity preserving surface: (a) $x z$-view and (b) $y z$-view for Figure 12, respectively.

Figure 2 shows the default bicubic Hermite spline for the positive data given in Table 3. Figures 3(a) and 3(b) show the $x z$-view and $y z$-view for Figure 2, respectively. Figure 4 shows the positivity preserving by using the proposed rational bicubic spline with $\alpha_{i, j}=\beta_{i, j}=0.5, \widehat{\alpha}_{i, j}=\widehat{\beta}_{i, j}=0.5$. Meanwhile, Figures 5(a) and 5(b) show the $x z$-view and $y z$ view for Figure 4, respectively.

Example 7. Positive data from the following function is truncated to five decimal places [1]:

$$
F_{2}(x, y)=\sin \left(y e^{-x}\right)+1, \quad-3 \leq x, y \leq 3, x, y \neq 0 .
$$

Figure 6 shows the default bicubic Hermite spline for the positive data given in Table 4. Figures $7(a)$ and $7(b)$ show the $x z$-view and $y z$-view for Figure 6, respectively. Figure 8 shows the positivity preserving by using the proposed rational bicubic spline with $\alpha_{i, j}=\beta_{i, j}=0.5, \widehat{\alpha}_{i, j}=\widehat{\beta}_{i, j}=0.5$. Meanwhile, Figures 9(a) and 9(b) show the $x z$-view and $y z$ view for Figure 8, respectively.

Example 8. Positive data from the following function is truncated to four decimal places [2]:

$$
F_{3}(x, y)=e^{-x^{2}}+e^{-2 y^{2}}+0.04, \quad-3 \leq x, y \leq 3 .
$$

Figure 10 shows the default bicubic Hermite spline for the positive data given in Table 5. Figures $11(\mathrm{a})$ and $11(\mathrm{~b})$ show the $x z$-view and $y z$-view for Figure 10, respectively. Figure 12 shows the positivity preserving by using the proposed rational bicubic spline with $\alpha_{i, j}=\beta_{i, j}=1.5, \widehat{\alpha}_{i, j}=\widehat{\beta}_{i, j}=1.5$. Meanwhile, Figures 13(a) and 13(b) show the $x z$-view and $y z$ view for Figure 12, respectively.

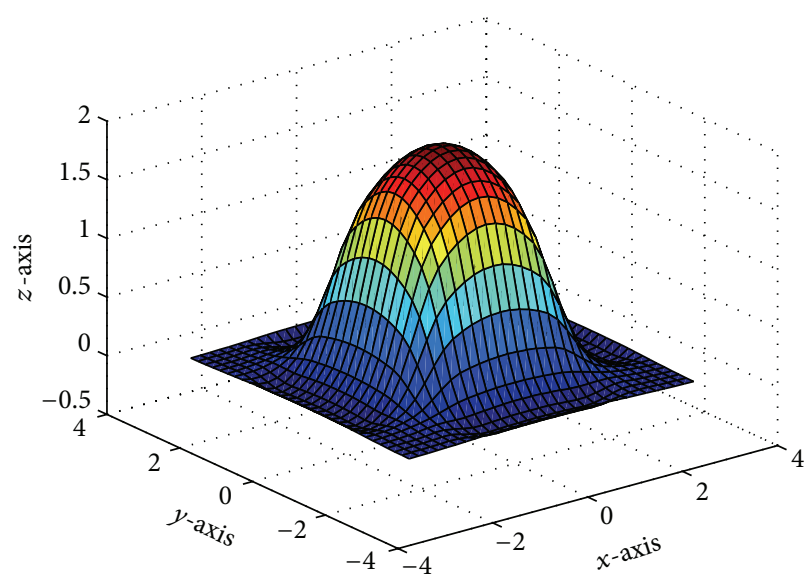

FIGURE 14: Bicubic Hermite surface.

Example 9. Positive data from the following function is truncated to four decimal places [12]:

$$
F_{4}(x, y)=\frac{4}{\left(\left(x^{2}+y^{2}\right)^{2}-1\right)}, \quad-3 \leq x, y \leq 3, x, y \neq 0 .
$$

Figure 14 shows the default bicubic Hermite spline for the positive data given in Table 6. Figures 15(a) and 15(b) show the $x z$-view and $y z$-view for Figure 14, respectively. Figure 16 shows the positivity preserving by using the proposed rational bicubic spline with $\alpha_{i, j}=\beta_{i, j}=1.5, \widehat{\alpha}_{i, j}=\widehat{\beta}_{i, j}=1.5$. Meanwhile, Figures 17(a) and 17(b) show the $x z$-view and $y z$ view for Figure 16, respectively. 


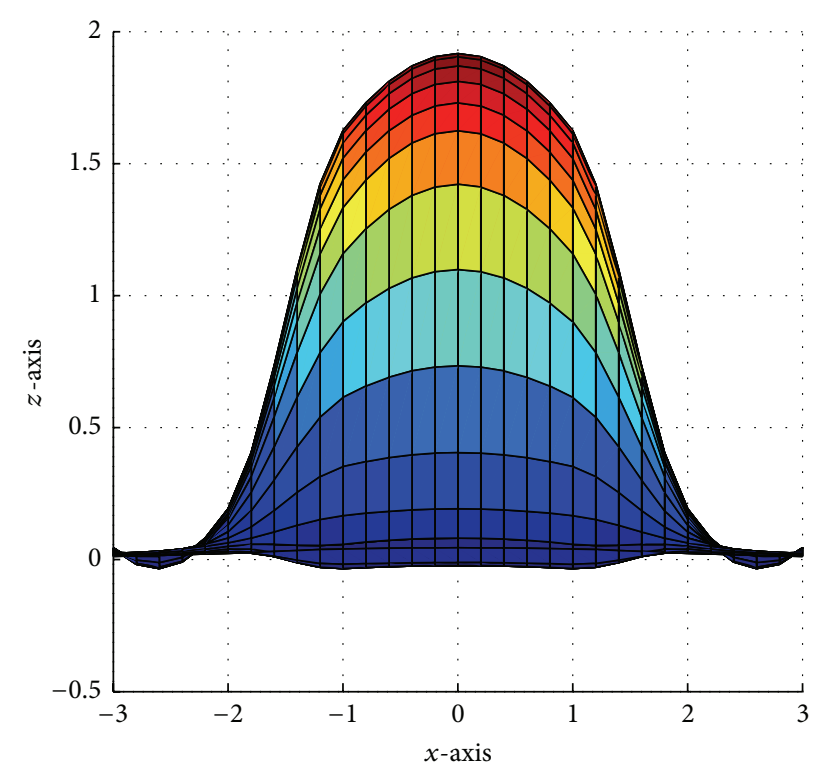

(a)

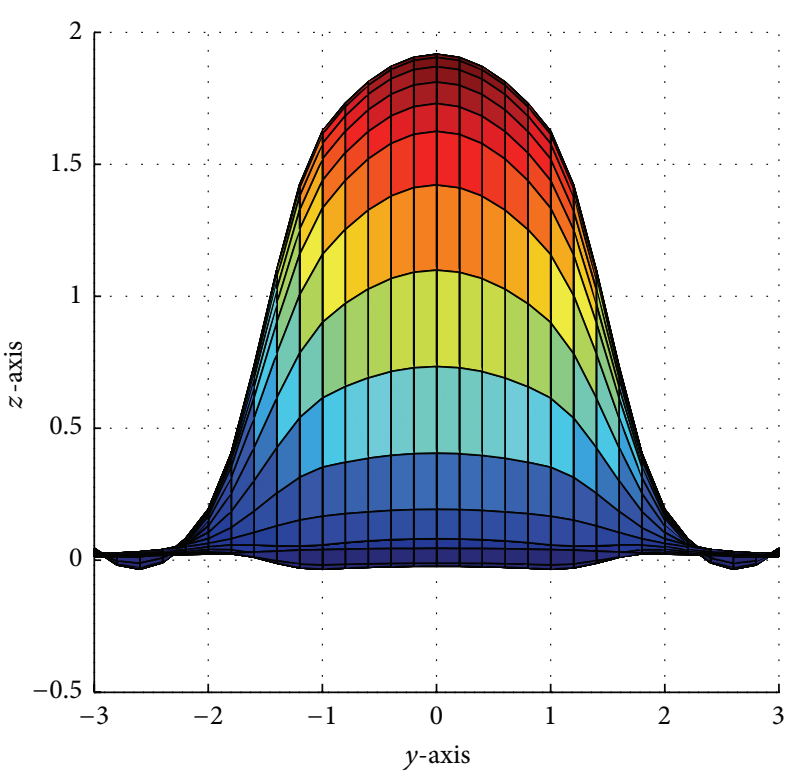

(b)

FIGURE 15: Bicubic Hermite surface: (a) $x z$-view and (b) $y z$-view for Figure 14, respectively.

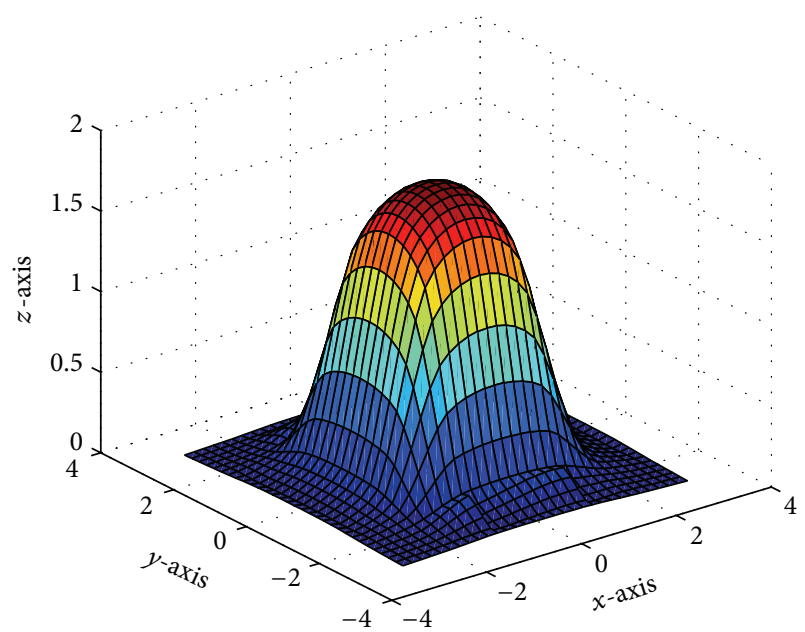

FIGURE 16: Positivity preserving by using our partially rational bicubic spline.

Meanwhile, Figures 18(a) and 18(b) show the positivity preserving by using the study by Abbas et al. [1] for positive data in Tables 3 and 4, respectively. Figure 19 shows the positivity preserving by using the study by Abbas et al. [2] for positive data in Table 5. Finally Figures 20 and 21 show the positive surfaces by using Hussain and Sarfraz [12] rational bicubic spline and Liu et al. [20] rational biquartic spline surfaces.

We compare the performance between our proposed partially blended rational bicubic spline interpolation and that of the work of Abbas et al. [1] by calculating Root Mean Square Error (RMSE). Table 7 summarized the results of RMSE estimation. It can be seen clearly that our rational bicubic spline interpolation is on a par with the work of Abbas
TABLE 7: RMSE estimation.

\begin{tabular}{lcccc}
\hline Method & \multicolumn{4}{c}{ Data sets } \\
& Example 6 & Example 7 & Example 8 & Example 9 \\
\hline Abbas et al. [1] & 0.3900 & 0.0252 & 0.0011 & 0.0103 \\
Ours & 0.3947 & 0.0233 & 0.0012 & 0.0106 \\
\hline
\end{tabular}

et al. [1]. The value for RMSE for all four data sets is almost the same. Thus we conclude that the proposed partially blended rational bicubic spline interpolation produces the positive surfaces through positive data sets with smaller RMSE. Furthermore we believe that the computation is less by using our rational bicubic spline interpolation compared with the other rational bicubic spline interpolation of Hussain and Sarfraz [12] and Sarfraz et al. [22].

\section{Discussions}

The proposed partially blended rational bicubic spline with 12 parameters has been used for positivity preserving of $3 \mathrm{D}$ positive data. The scheme has been tested on 4 different data sets. The bicubic Hermite spline does not have the capability to preserve the positivity of the data without changing the original data points. Figures 2, 6, 10, and 14 show that there exist some negative values when the data sets are interpolated by using bicubic Hermite spline. From Figures 4, 8, 12, and 16 it can be seen clearly that the proposed scheme gives comparable results with existing schemes such as those of Abbas et al. [1, 2], Hussain and Sarfraz [12], and Liu et al. [20]. Similar to the work of Abbas et al. [1], our scheme also is partially blended bicubic rational spline. Meanwhile, Abbas et al. [2] and Hussain and Sarfraz [12] have used rational bicubic 


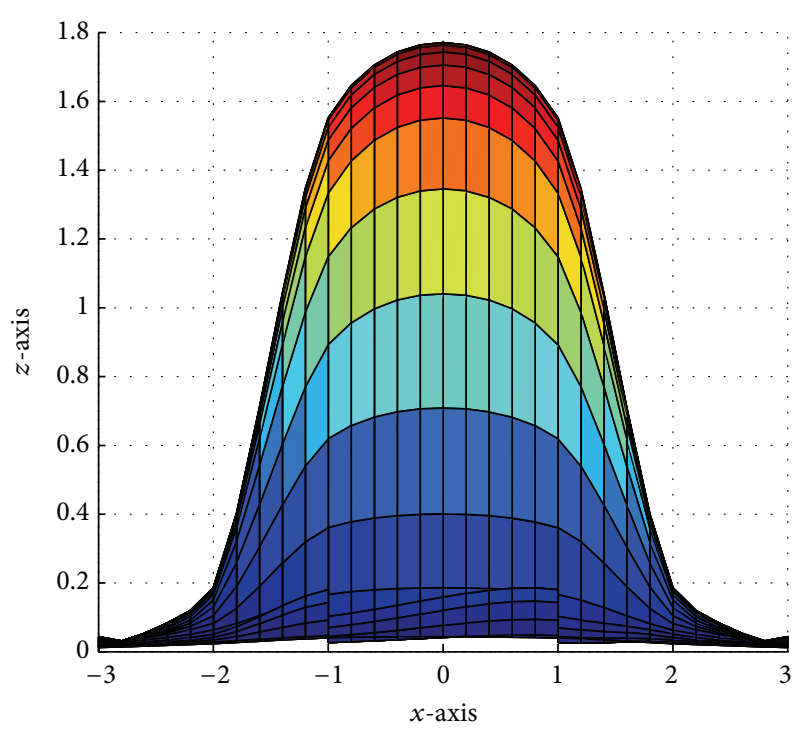

(a)

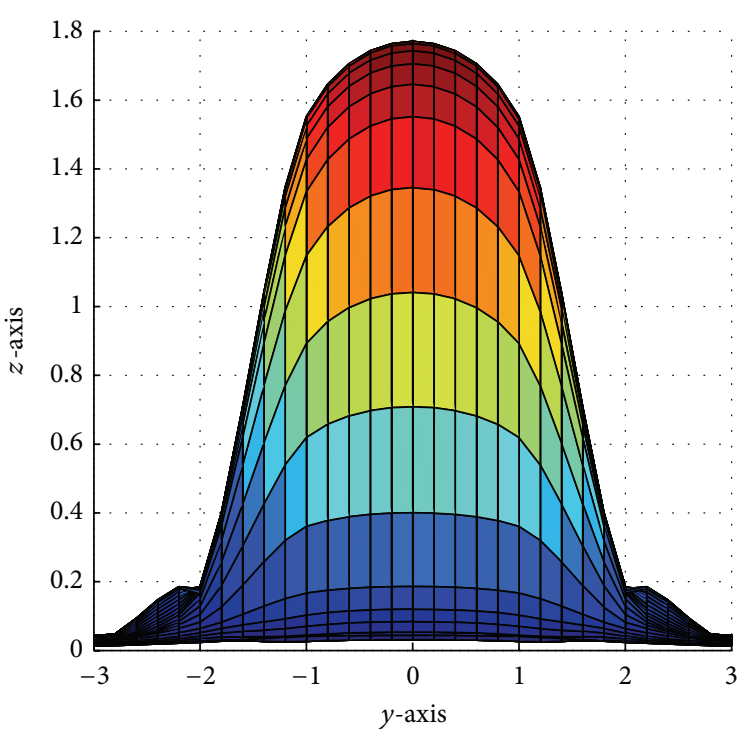

(b)

FIGURE 17: Positivity preserving surface: (a) $x z$-view and (b) $y z$-view for Figure 16, respectively.

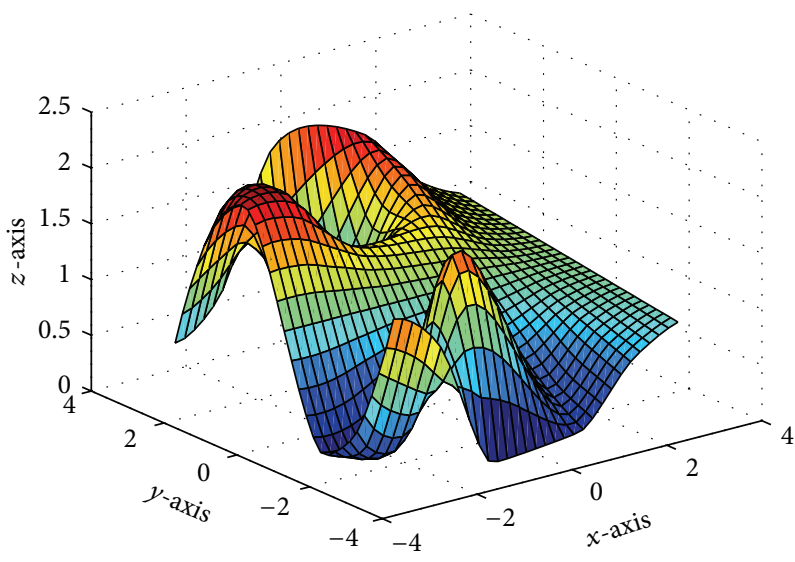

(a)

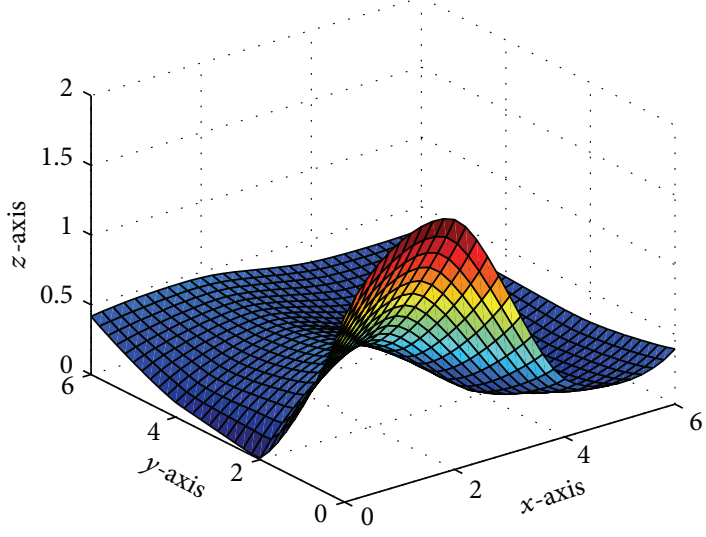

(b)

FIgURE 18: Positivity preserving by using the study of Abbas et al. [1] for (a) positive data in Table 3 and (b) positive data in Table 4.

spline interpolation. Table 7 summarized the RMSE values comparison between the proposed partially blended rational bicubic spline interpolation and that of the work of Abbas et al. [1].

\section{Summary and Conclusions}

In this paper the rational cubic spline of Karim and Kong $[18,19]$ has been extended to the bivariate cases. The partially blended bicubic rational spline has 12 parameters in the descriptions and 8 of them are free parameters. These free parameters can be used to change the shape of the final surfaces of the positive data. Graphical comparison between the proposed schemes and existing schemes also has been done. From all numerical results, we concluded that the proposed partially blended bicubic rational spline works well for all tested data sets. Future works will be developing the rational bicubic spline for scattered data interpolation without the need to modify the first derivative. This will improve the work of Peng et al. [26] that requires the modification of the first derivative in which positivity preserving is not met. Work on monotonicity and convexity preservation by using the proposed partially blended rational bicubic spline interpolation is underway.

\section{Conflict of Interests}

The authors declare that there is no conflict of interests regarding the publication of this paper. 


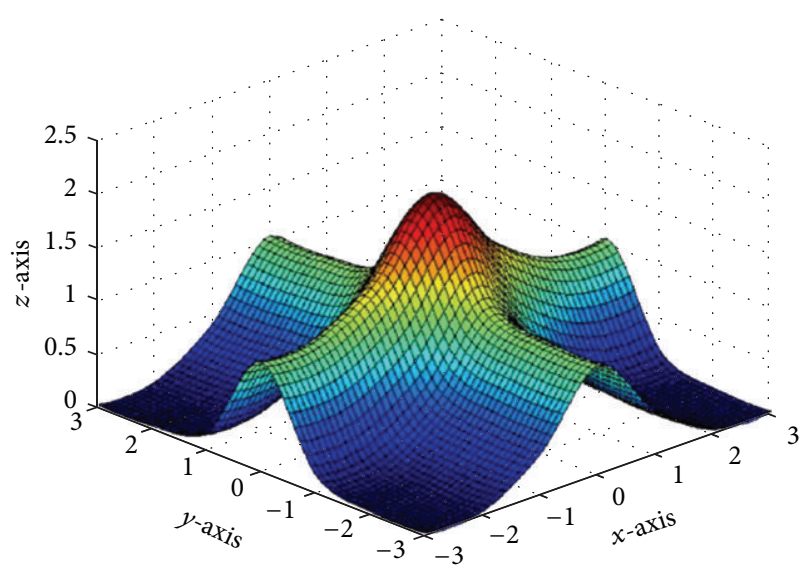

FIGURE 19: Positivity preserving by using the study of Abbas et al. [2] for positive data in Table 5.

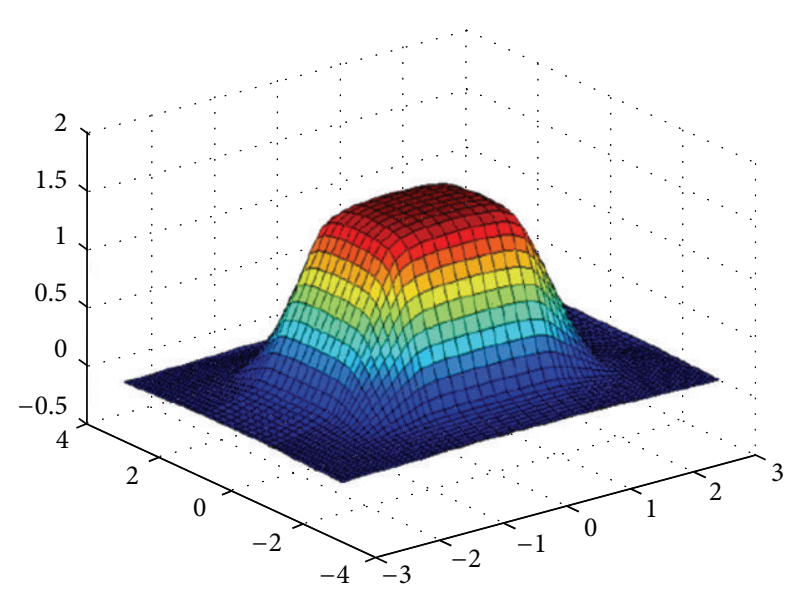

FIGURe 20: Positivity preserving by using the study of Hussain and Sarfraz [12] for positive data in Table 6.

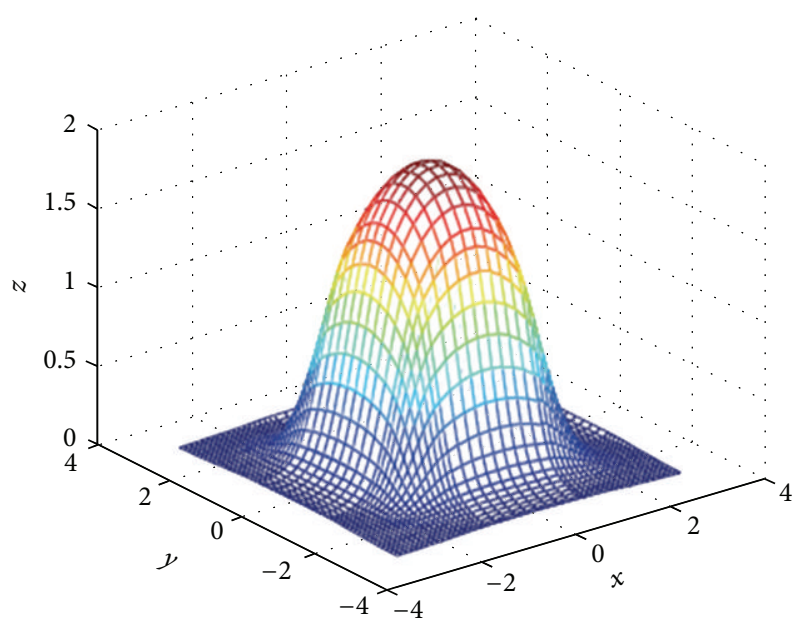

FIGURe 21: Positivity preserving by using the study of Liu et al. [20] for positive data in Table 6 .

\section{Acknowledgment}

The first author would like to acknowledge Universiti Teknologi PETRONAS (UTP) for the financial support received in the form of a research grant: Short Term Internal Research Funding (STIRF) no. 35/2012 including the Mathematica Software.

\section{References}

[1] M. Abbas, A. A. Majid, and J. M. Ali, "Shape preserving rational bi-cubic function for positive data," World Applied Sciences Journal, vol. 18, no. 11, pp. 1671-1679, 2012.

[2] M. Abbas, A. A. Majid, and J. M. Ali, "Positivity-preserving rational bi-cubic spline interpolation for 3D positive data," Applied Mathematics and Computation, vol. 234, pp. 460-476, 2014.

[3] M. R. Asim and K. W. Brodlie, "Curve drawing subject to positivity and more general constraints," Computers \& Graphics, vol. 27, no. 4, pp. 469-485, 2003.

[4] S. Butt and K. W. Brodlie, "Preserving positivity using piecewise cubic interpolation," Computers and Graphics, vol. 17, no. 1, pp. 55-64, 1993.

[5] K. W. Brodlie, P. Mashwama, and S. Butt, "Visualization of surface data to preserve positivity and other simple constraints," Computers and Graphics, vol. 19, no. 4, pp. 585-594, 1995.

[6] M. R. Asim, G. Mustafa, and K. W. Brodlie, "Constrained visualization of $2 \mathrm{D}$ positive data using modified quadratic Shepard method," in Proceedings of the International Conference in Central Europe on Computer Graphics and Visualization (WSCG '04), vol. 27, pp. 469-485, Plzeň, Czech Republic, February 2004.

[7] Q. Duan, L. Wang, and E. H. Twizell, "A new bivariate rational interpolation based on function values," Information Sciences, vol. 166, no. 1-4, pp. 181-191, 2004.

[8] Q. Duan, H. Zhang, Y. Zhang, and E. H. Twizell, "Bounded property and point control of a bivariate rational interpolating surface," Computers \& Mathematics with Applications, vol. 52, no. 6-7, pp. 975-984, 2006.

[9] Q. Duan, F. Bao, and Y. Zhang, "Shape control of a bivariate interpolating spline surface," International Journal of Computer Mathematics, vol. 85, no. 5, pp. 813-825, 2008.

[10] T. N. T. Goodman, B. H. Ong, and K. Unsworth, "Constrained interpolation using rational cubic splines," in Proceedings of the NURBS for Curve and Surface Design, G. Farin, Ed., pp. 59-74, 1991.

[11] T. N. T. Goodman, "Shape preserving interpolation by curves," in Algorithms for Approximation IV, J. Levesley, I. J. Anderson, and J. C. Mason, Eds., pp. 24-35, University of Huddersfield Proceedings, Huddersfield, UK, 2002.

[12] M. Z. Hussain and M. Sarfraz, "Positivity-preserving interpolation of positive data by rational cubics," Journal of Computational and Applied Mathematics, vol. 218, no. 2, pp. 446-458, 2008.

[13] M. Z. Hussain and M. Hussain, "Visualization of data subject to positive constraint," Journal of Information and Computing Sciences, vol. 1, no. 3, pp. 149-160, 2006.

[14] M. Z. Hussain and M. Hussain, " $C^{1}$ positive scattered data interpolation," Computers \& Mathematics with Applications, vol. 59, no. 1, pp. 457-467, 2010.

[15] M. Tian, Y. Zhang, J. Zhu, and Q. Duan, "Convexity-preserving piecewise rational cubic interpolation," Journal of Information and Computational Science, vol. 2, no. 4, pp. 799-803, 2005. 
[16] M. Z. Hussain, M. Sarfraz, and A. Shakeel, "Shape preserving surfaces for the visualization of positive and convex data using rational biquadratic splines," International Journal of Computer Applications, vol. 27, no. 10, pp. 12-20, 2011.

[17] F. Ibraheem, M. Hussain, M. Z. Hussain, and A. A. Bhatti, "Positive data visualization using trigonometric function," Journal of Applied Mathematics, vol. 2012, Article ID 247120, 19 pages, 2012.

[18] S. A. A. Karim and V. P. Kong, "Local control of the curves using rational cubic spline," Journal of Applied Mathematics, vol. 2014, Article ID 872637, 12 pages, 2014.

[19] S. A. A. Karim and V. P. Kong, "Shape preserving interpolation using rational cubic spline," Research Journal of Applied Sciences, Engineering and Technology, vol. 8, no. 3, pp. 312-320, 2014.

[20] X. Liu, Y. Zhu, and S. Liu, "Positivity and monotonicity preserving biquartic rational interpolation spline surface," Journal of Applied Mathematics, vol. 2014, Article ID 987076, 11 pages, 2014.

[21] A. Saaban, A. A. Majid, and A. R. Piah, "Visualization of rainfall data distribution using quintic triangular Bezier patches," Bulletin of the Malaysian Mathematical Sciences Society, vol. 32, no. 2, pp. 137-150, 2009.

[22] M. Sarfraz, M. Z. Hussain, and A. Nisar, "Positive data modeling using spline function," Applied Mathematics and Computation, vol. 216, no. 7, pp. 2036-2049, 2010.

[23] M. Bastian-Walther and J. W. Schmidt, "Range restricted interpolation using Gregory's rational cubic splines," Journal of Computational and Applied Mathematics, vol. 103, no. 2, pp. 221-237, 1999.

[24] J. A. Gregory, "Shape preserving spline interpolation," Computer-Aided Design, vol. 18, no. 1, pp. 53-57, 1986.

[25] J. Wu, X. Zhang, and L. Peng, "Positive approximation and interpolation using compactly supported radial basis functions," Mathematical Problems in Engineering, vol. 2010, Article ID 964528, 10 pages, 2010.

[26] X. Peng, Z. Li, and Q. Sun, "Nonnegativity preserving interpolation by $C^{1}$ bivariate rational spline surface," Journal of Applied Mathematics, vol. 2012, Article ID 624978, 11 pages, 2012.

[27] G. E. Farin, Curves and Surfaces for CAGD: A Practical Guide, Morgan Kaufmann Publishers, San Francisco, Calif, USA, 5th edition, 2002.

[28] M. Z. Hussain, M. Sarfraz, and T. S. Shaikh, "Shape preserving rational cubic spline for positive and convex data," Egyptian Informatics Journal, vol. 12, no. 3, pp. 231-236, 2011.

[29] G. Casciola and L. Romani, "Rational interpolants with tension parameters," in Curve and Surface Design, T. Lyche, M.-L. Mazure, and L. L. Schumaker, Eds., pp. 41-50, 2003. 


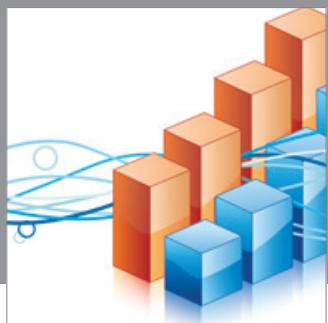

Advances in

Operations Research

mansans

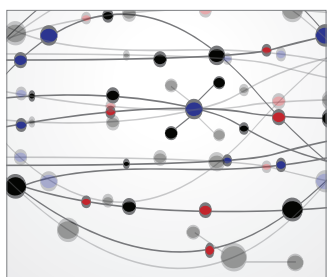

The Scientific World Journal
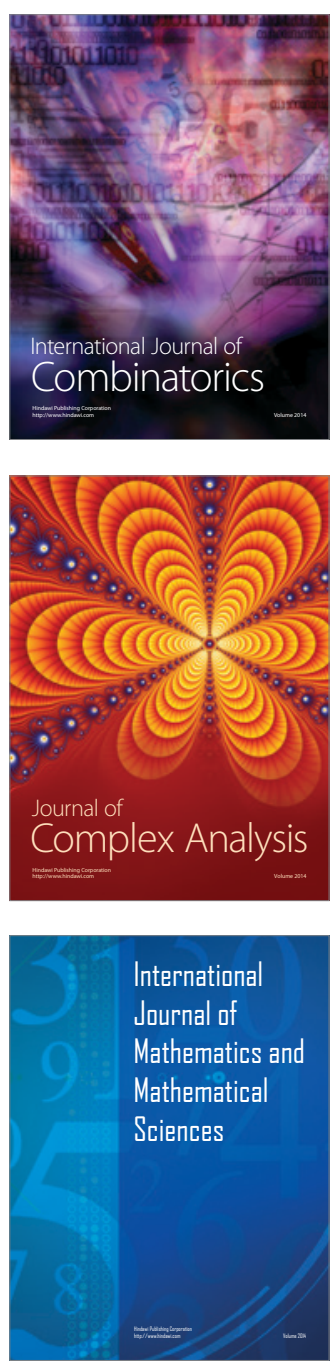
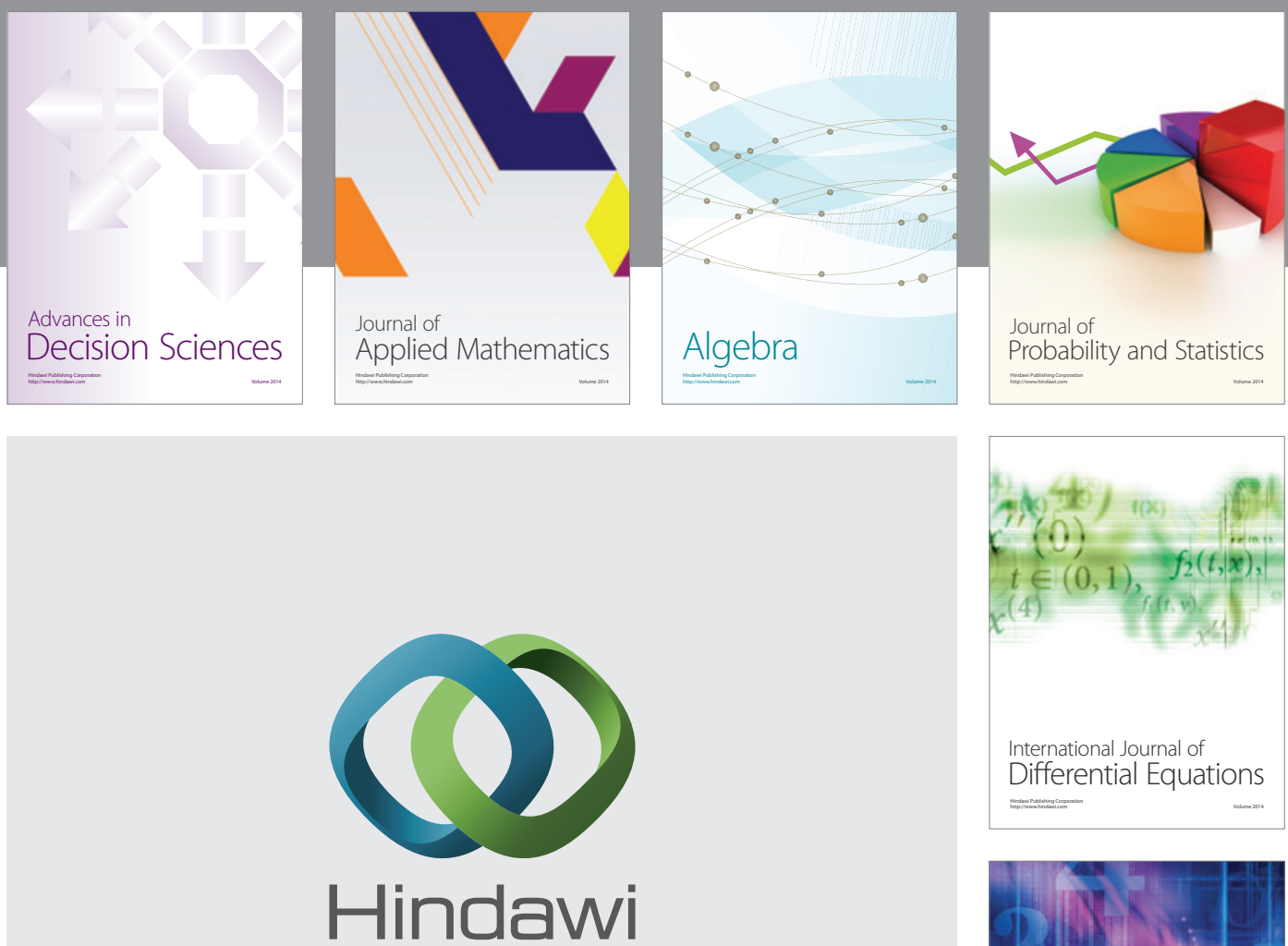

Submit your manuscripts at http://www.hindawi.com
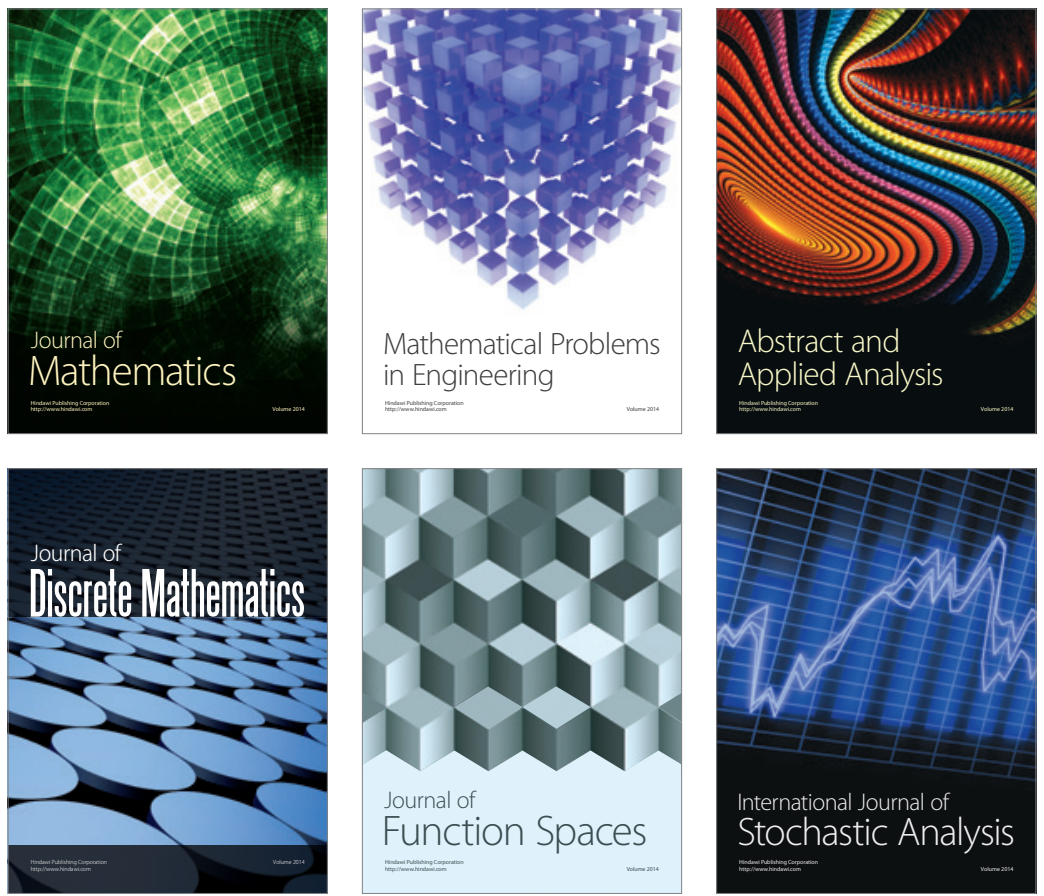

Journal of

Function Spaces

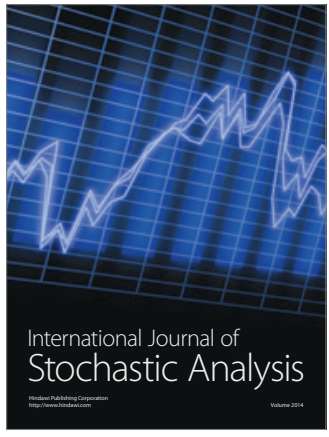

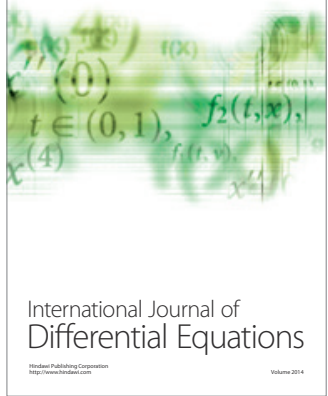
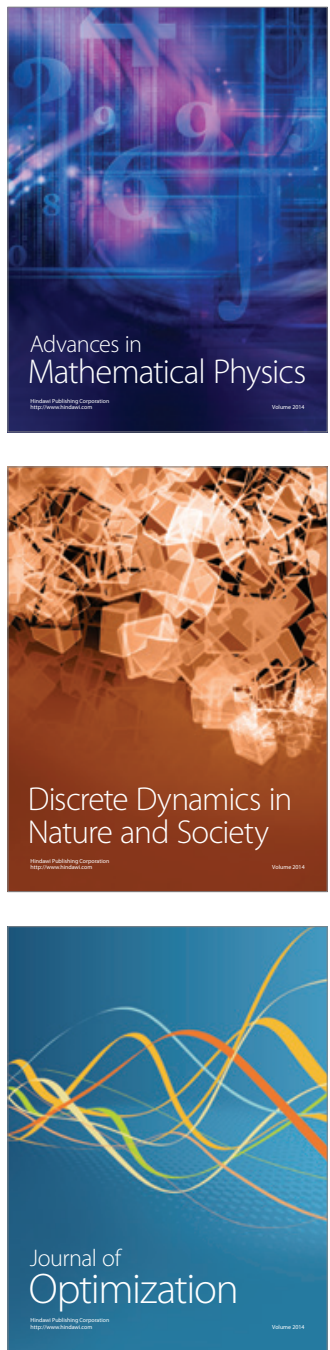